\title{
Advanced Design and Fabrication of Microwave Components Based on Shape Optimization and 3D Ceramic Stereolithography Process
}

\author{
N. Delhote ${ }^{1}$, S. Bila ${ }^{1}$, D. Baillargeat ${ }^{1}$, T Chartier $^{2}$ and S Verdeyme ${ }^{1}$ \\ ${ }^{1}$ XLIM, UMR 6172, Université de Limoges/CNRS \\ 2SPCTS UMR 6638, Université de Limoges/CNRS \\ Limoges, \\ France
}

\section{Introduction}

The design of advanced components for space and terrestrial telecommunication systems requires both sophisticated design methodologies and manufacturing technologies for improving current component characteristics. In particular, optimizing the shape and the size of a component is a problem of primary importance for microwave engineers. Moreover, for designing RF and microwave components or antennas, the use of ceramic materials is preferable in order to satisfy both electrical and dimensional constraints.

The main objective of this chapter is to demonstrate that it is possible to jointly improve the design and fabrication procedures of ceramic based advanced RF components. In this context, a ceramic 3D stereolithography based rapid prototyping technique is applied for fabricating 3D ceramic structures. As presented next, theoretical and experimental approaches are complementary and innovative components with excellent electrical performances have been designed, manufactured and characterized. Then the contribution demonstrates how an original CAD design approach based on shape optimization methods can be applied for improving electrical performance and integration of microwave and millimeter-wave devices.

The chapter is organized as follows:

- In the first section of this chapter we will focus on the explanation of the ceramic 3D stereolithography (SLA) process. A particular attention on the process developed by the SPCTS UMR 6638 (Limoges, France) and now available at the Centre de Transfert de technologies Ceramiques (CTTC, Limoges, France) will be paid here. This process has been developed in recent years, in the context of rapid prototyping techniques. More generally three dimensional (3-D) fabrication processes have recently emerged as enabling technologies for the design of compact devices and interconnects, and for packaging needs. For these purposes, polymer (Liu, 2004) and ceramic (Brakora et al., 2007; Delhote et al,2007) stereolithography methods are really promising since they do not require the use of moulds and cutting tools. They are relatively fast and reliable techniques capable of building truly 3-D structures with a high aspect ratio and accuracy. 
- The second section is devoted to the description of several test structures. As demonstrated here, the applications concern several areas of microwave engineering, from very complex 3D periodic structures, to advanced millimeter wave antenna and bandpass filters. All the devices were designed applying a 3D electromagnetic software, and were fabricated and tested successfully. The satisfying agreement between theoretical and experimental studies allows us to validate the use of our rapid prototyping technique for fabricating millimeter wave devices.

- The third section is dedicated to the description of shape optimization methods that can be used for computer-aided design of microwave components. Two techniques have been implemented and coupled to an electromagnetic solver based on a finite element method and have been used successfully for the design of ceramic-based microwave components. Several examples are described.

\section{3D ceramic stereolithography process}

\subsection{Rapid prototyping process}

The stereolithography process, commonly referred as layer-by-layer or SLA, can be attached to a more general class of process called rapid prototyping process. One general definition of the fast prototyping fabrication technique is a manufacturing process capable of producing a prototype with a minimum of time and resource (no need for mould for example). The SLA process is an additive process in comparison with traditional substractive shaping techniques like cutting, milling or drilling. The principle of this manufacturing technique can be summarized in Figure 2.1.

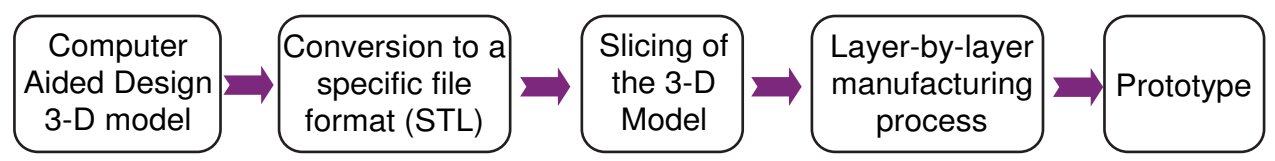

Fig. 2.1. Principle of a layer-by-layer manufacturing process

The starting point of this fabrication chain is the creation of the initial drawing with a Computer Aided Design (CAD) software. This drawing represents the future prototype to build. Then, a conversion software is applied to convert this model into a very specific format called STL. Initially developed for the stereolithography process, it has become the standard format for additive process. This format turns the 3-D model surface into a set of triangles, a rather simple geometry to work with. Then the 3D-model, once converted, is sliced into fixed height slices. The core of this kind of manufacturing technique is to build slice after slice ("layer-by-layer") the whole 3-D objet. The fabrication can now begin and the prototype, with the geometry and dimensions defined in the initial CAD model is finally retrieved.

One can mention the most common 3-D additive techniques like the Selective Laser Sintering (SLS) and a version more dedicated to the fabrication of metal and ceramic parts called Direct Metal Laser Sintering (DMLS), the Laminated Object Manufacturing (LOM) process, Fused Deposition Modelling (FDM), 3D Printing (3DP) and the Inkjet Printing. Among theses techniques, the stereolithography has been the first and most common $3 \mathrm{D}$ manufacturing process for the creation of prototypes. 


\subsection{Stereolithography}

The principle now described is the most widely used by research teams and commercial devices. As mentioned earlier, the point of this process is to build the slices of the 3D model one by one, from the bottom of the object to its top. It is based on the polymerisation of a photosensitive resin by a laser, most of the time being in the Ultraviolet wavelength range (Hull, 1986). The Figure 2.2 gives an overview of the device.

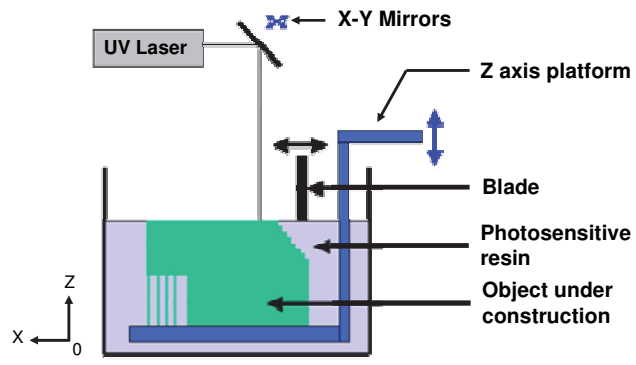

Fig. 2.2. Principle of the SLA manufacturing process

The resin disposed in a tank needs to be very fluid and is composed of photosensitive monomers. These elements can turn into polymers if enough energy is given through a laser beam and constitutes the very heart of 3D additive process (Hull, 1986). Indeed, once polymerised, the resin is quite solid and can be easily separated from the liquid monomers. In the tank containing the photosensitive resin, a platform moving along the vertical $(\mathrm{z})$ axis is placed in order to support the part during its fabrication. Its position is monitored by a computer with a very high positioning accuracy (typically a few micrometers).

For the fabrication of the first slice (layer) of the object, the platform is initially place close to the surface of the resin: the distance between the surface and the platform is more precisely equal to the thickness of the slice. A blade, also monitored by a computer, then goes at a defined speed along the surface of the liquid to make it as flat as possible.

The surface of the liquid will now be selectively exposed to the UV laser thanks to two mobile mirrors according to the pattern of the first slice. These mirrors are illuminated by a fixed laser beam and monitored by computer. With this process, the slice of the object is physically drawn at the surface with the laser polymerising the resin on its path. The polymerisation reaction depth (see Figure 2.3) is also dependant on the amount of power transported by the laser beam.

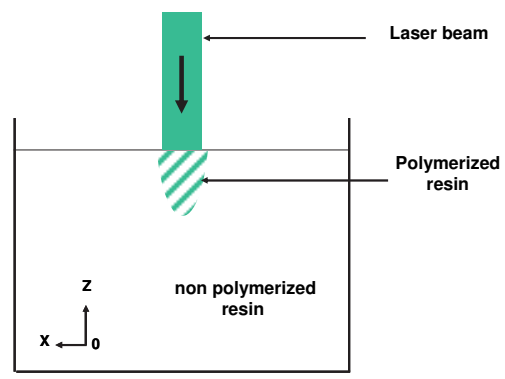

Fig. 2.3. Thickness of polymerisation 
The laser beam is slightly scattered at the surface of the resin because of the refractive index difference between the air and the resin. Thus, the laser spot at the resin surface is larger than the initial beam diameter.

After the first slice is done, the $\mathrm{Z}$ axis platform goes down with a distance equal to the object second slice thickness and the process is repeated until all the slices are completed.

As seen on Figure 2.3, the polymerised amount of resin has an oblong shape with a diameter becoming smaller as the polymerising depth goes higher. The energy transported by the laser beam has to be very precisely calibrated because it directly defines the thickness of polymerised resin (Chartier, 2002). Indeed, the power transported by the laser beam has to be enough to trigger the polymerisation process but also has to polymerise a sufficient thickness to make the slice under construction attached to the previous layer. If not, the slices are not glued together and the whole part will be delaminated.

At the end of the process, the fabricated part, i.e. the solid polymerised part can be easily retrieved from the liquid resin bath. If needed, a post UV curing can be done in order to fully polymerise the whole part. The accuracy of this process is mainly driven the diameter of the laser beam and very high accuracy close to $5 \mu \mathrm{m}$ can achieved with the appropriate laser (Monneret, 2005).

Many research teams have then tried to use this technique to manufacture 3D object made out of ceramic materials (Bertsch, 2003) by adding a volume percentage of ceramic particles into the photosensitive resin. The term ceramic suspension or paste is in this case more appropriate.

\subsection{Evolution to ceramic stereolithography}

In order to use the process describe in 2.2, the resin, now with ceramic particles, has to remain very fluid. If the compound is too viscous, the blade cannot ensure anymore a proper flatness at the surface of the resin bath and critical deformations are observed during fabrication. To do so, the logical way is to limit the total volume percentage of ceramic particles because the overall viscosity of the paste is directly related to this parameter (Hinczewski, 1998). However in order to obtain ceramic objects with appropriate properties et mechanical strength and to limit cracks during the necessary firing step, the volume percentage of ceramic particles has to be more than $50 \%$ in volume (Hinczewski, 1998).

There is here a deep contradiction because for high percentage, the compound is very viscous. This very high viscosity can also lead to a very high UV exposition time to trigger the polymerisation reaction. In certain circumstances, the critical energy required to polymerise the compound can even not be reached.

Based on such considerations, Thierry Chartier (SPCTS UMR 6638, Limoges, France) in collaboration with the technological transfer centre CTTC (Centre de Transfert de Technologies Ceramiques, Limoges, France) proposed a different approach (Doreau, 2000). Instead of developing a mix, called more specifically a suspension, which should be as fluid as possible, $\mathrm{T}$. Chartier has developed a suspension with a high volume percentage $(\sim 65 \%)$ of ceramic particles and highly selective photosensitive resin (Doreau, 2000). The obtained suspension is in such configuration thick and viscous.

This composition has then been used by many research teams (Bertsch, 2003).

\subsection{Preparation of a ceramic suspension}

Adding such amount of ceramic particles in the suspension increases the complexity of its preparation. As its quality is directly dependant on the homogeneous mixing of all its chemicals elements, a very precise protocol has to be followed. 
The first step is to sift the ceramic powder in order to keep only particles with a very precise diameter from one to a few micrometers. Their diameter, size distribution and geometry have to be precisely controlled because they directly have an impact on the suspension properties and those of the ceramic part which will be made (Hinczewski, 1998).

These ceramic particles naturally tend to agglomerate together and this behaviour has to be avoided as much as possible. Thus the ceramic particles are mixed with ethanol and a dispersal agent. This latter modifies the interaction forces between the particles by playing on electrostatic repulsion forces for example. Thanks to them, a global homogeneity is ensured within the suspension. This mix is then grinded by highly hard ceramic balls from less than one hour to many hours.

After this grinding step, the mix is placed in an oven to evaporate the ethanol. The obtained very thin particles are then mixed with other elements:

- A photosensitive resin composed of reactive monomers which can be polymerised under UV light exposition

- A photo-initiator which absorbs the UV light and helps the polymerisation process of the resin

- A binder: the goal of this chemical is the keep the particles together enough to ensure a mechanical strength of the ceramic part before the firing step. This part is called a green part.

- A plasticizer which helps the green part to be rigid enough to endure the fabrication process

- A fluidifier which brings the suspension fluidity to a required level

- A wetting agent which helps to control the adhesion between the powder and the binder, making the whole suspension homogeneous

The obtained suspension then goes through rollers to break agglomerates if they still exist. After that the obtained viscous paste has to remain still for some hours for its outgassing and is now ready to be used for the manufacturing steps.

Because of its high viscosity, a very specific sweeping system has to be used with it.

\subsection{Ceramic stereolithography manufacturing process}

The next Figure 2.4 shows the manufacturing platform of the stereolithography process.

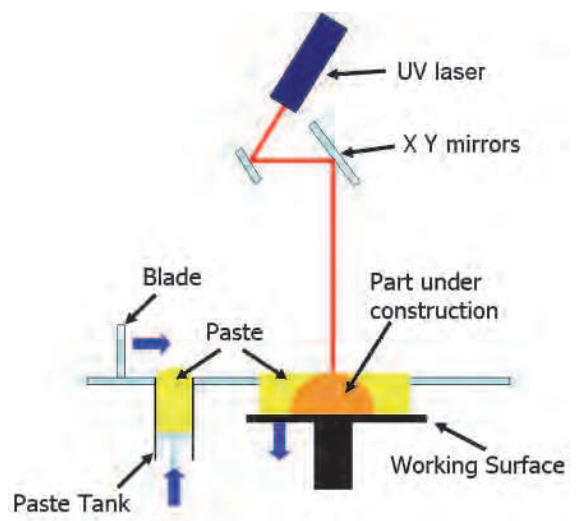

Fig. 2.4. Principle of the stereolithography 3D process 
This technique shares many common points with the standard stereolithography process described in part 2.1.

A Ultraviolet (UV) $(\lambda \sim 350 \mathrm{~nm})$ laser is still commonly used to selectively polymerise layers of the prepared suspension to build a 3D object. However because of the high viscosity of the paste used for the fabrication, the manufacturing device needs some modifications.

The paste is put in a paste fed container and a piston delivers the right amount of paste for one layer. Then a specifically profiled blade spreads uniformly the paste on the working surface.

After the first layer is spread, (10 to 15 seconds), this latter is selectively exposed to the UV laser beam thanks to X-Y monitored mirrors within seconds to physically draw the first slice of the 3-D object. The typical diameter of the beam is about $100 \mu \mathrm{m}$. After that, the working surface goes down for a distance equal to the thickness of a layer. Another layer of paste is then spread at the top of the previous one and the process continues in the same way for every layer.

The working surface of the device used to manufacture the ceramic parts presented in part 2 is $25 \mathrm{~cm}$ by $25 \mathrm{~cm}$. The maximum height which can be obtained is also $25 \mathrm{~cm}$. For a $1 \mathrm{~cm}$ thick part composed of $100 \mu \mathrm{m}$ thick layers, the manufacturing time is close to 1 hour. Thinner layers of $25 \mu \mathrm{m}$ can be reached with this system.

At the end of the fabrication, because of the paste viscosity, the polymerised part has to be retrieved from the amount of non-polymerised paste. The part is then cleaned by going through different chemical baths. Because of the cleaning step, the part can not have closed volumes and if the part is closed, it needs at least holes to evacuate the non-polymerised paste.

The obtained cleaned part is called the green part and will need to be fired to obtain its final dimensions and properties. Because of the manufacturing process specificities, the suspension has to show specific properties.

\subsection{Properties of the suspension}

\subsubsection{Mechanical strength}

The paste has to be viscous enough to be correctly spread by the blade on top of working surface. The spread layer has to keep a defined thickness and a uniform aspect during the process. Because of the blade, a meniscus grows at its bottom but this phenomenon can be limited by refining the suspension composition. Thus, no extra time between the spreading of each layer is required to let the meniscus disappear. The suspension used by Chartier et al. (Chartier 2002) is about 10 times more viscous than the suspension used by other research teams (Bertsch, 2003). This high viscosity as explained in part 2.4 is mainly due to the high volume percentage of ceramic particles. Thanks to this viscosity, a layer of non-polymerised paste can generally support the other layers spread on top of it.

However in some cases in order to avoid deformations of the part because of the blade movement, it can be necessary to add some construction supporting elements. These elements are polymerised parts made during the manufacturing. A typical case is shown in the next Figure 2.5.

Typically some pillars acting as supporting elements can be added in critical points where the main part required extra strength during the fabrication process. 


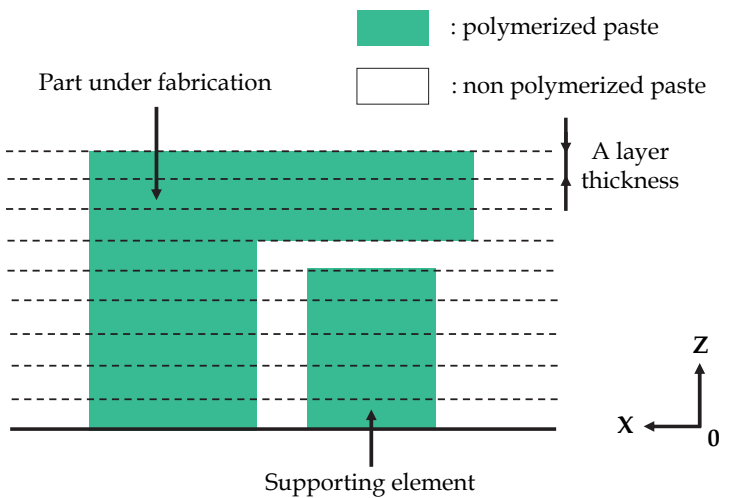

Fig. 2.5. Supporting elements added during manufacturing of the main part

\subsubsection{Light scattering and absorption}

Adding ceramic particles in the initial photosensitive resin modifies the thickness and width of the polymerised zone exposed to the UV laser beam. The ceramic particles indeed create a combined light scattering and absorption phenomenon as shown in Figure 2.6.

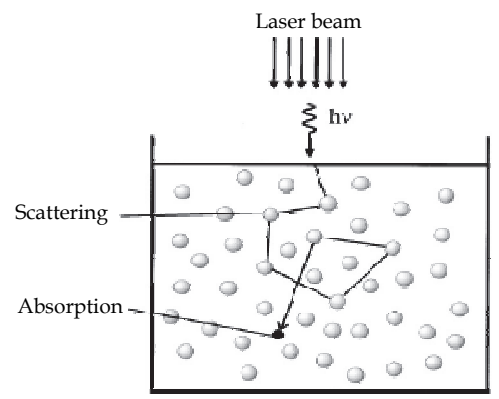

Fig. 2.6. Light scattering and absorption phenomena in a media charged in ceramic particles

The laser beam spot at the surface of the suspension layer is thus 1.5 to 2.5 times wider than its original width. The resolution obtained is consequently lower than with a non charged resin and typical manufacturing tolerances are about $100 \mu \mathrm{m}$. This value can however be decreased with refinements on every aspects of the process.

The ceramic particles also absorb a part of the energy provided by the laser beam and tend to limit the polymerisation thickness which will be called $E p$ in the equation 2.1.

$$
E_{p}=D_{p} \ln \left(\frac{D E}{D E_{C}}\right) \text { in meters }
$$

$D E$ is the energy density given to the resin defined by the equation 2.2.

$$
D E=\frac{2 P}{\pi \cdot w_{0} \cdot v} \text { in } \mathrm{J} \cdot \mathrm{m}^{2}
$$


With $P$, the insulation power (in $\mathrm{J}^{-1}{ }^{-1}$ ), $w_{0}$ the radius of the laser beam in meters and $v$ the

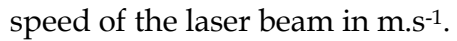

$D E_{c}$ is the critical energy density which is the minimal energy to trigger the polymerisation process in $\mathrm{J}^{\mathrm{m}} \mathrm{m}^{2}$.

$D p$ is finally the laser beam depth of penetration in meters.

The polymerisation thickness $E p$ is thus directly proportional to the depth of penetration of the laser beam $D p$. For suspension charged with ceramic particles, $D p$ is defined by the equation 2.3:

$$
D_{p}=\frac{2}{3} \frac{d_{50}}{Q . \phi} \text { in meters }
$$

with $d_{50}$ the average diameter of the particles in meters, $\phi$ the volume percentage of particles and $Q$ is a coefficient defined by equation 2.4 :

$$
Q=\frac{h}{\lambda} \cdot \Delta n^{2}
$$

with $h$ the average inter-particular distance in meters, $\lambda$ the wavelength of the laser in meters and $\Delta n^{2}$ the difference between the refractive index of the ceramic powder and the resin defined by the equation 2.5 :

$$
\Delta n^{2}=\left(n_{\text {powder }}-n_{r e \sin }\right)^{2}
$$

The higher the ceramic particles percentage is the less the laser beam depth of penetration is. In the same way, high refractive index ceramic powders or low diameter ceramic particles can also strongly decrease this penetration depth.

The equation 1 also indicates that the less $D p$ is, the less Ep will be.

Remembering the shape of the polymerised paste under the action of the laser (Figure 2.3), $D p$ has also a significant impact on the polymerization width at the surface of a layer of paste. The higher $D p$ is the less the lateral definition will be. A compromise has thus to be find between a polymerisation depth high enough to fully polymerised a layer thickness but not too high to not lose too much lateral definition.

As shown in (Chartier, 2002), there is a relation between the polymerisation depth (and lateral definition) and the concentration of the photo-initiator in the suspension. High concentration levels of this element indeed increase the energy density received by the paste without a need of high $D p$ values. Refining the photo-initiator concentration level can thus lead to the required polymerisation depth without sacrificing the definition.

At the end, the concentration of every constituent in the suspension has to be finely defined to obtain the right compromise between high volume percentage of ceramic particles, acceptable definition and polymerisation depth.

\subsubsection{Shrinkage during firing}

In the previous part it has been seen that high volume percentages of ceramic particles within the suspension decrease the definition of the stereolithography process. However these high levels of concentration are highly interesting because they will help to decrease the ceramic part deformations during its fabrication. Indeed, the green part retrieved at the end of the process has to go through two final firing steps which will put more mechanical stress on it. 
The first firing step is called debinding. This step will burn out organic materials which are still in the part like the polymers, binders... The part is fired while constantly increasing the temperature up to about $600^{\circ} \mathrm{C}$. This last temperature is then maintained for a few hours. During this step, the organic elements within the green part slowly move to the outside of the part. Deformations and even cracks can appear if the temperature goes too fast to the maximum value. The temperature has to be increased very slowly, typically less than a Celsius degree per minute.

During the second step, the temperature keeps increasing up to more than $1600^{\circ} \mathrm{C}$ with a higher ramp of many Celsius degrees per minute. This step called sintering gives the ceramic part its final density with typical values equal or more than $97 \%$ of the theoretical value. The part finally presents its final density and size.

During these two steps, the green part shrinks and the shrinkage percentage is directly related to the volume percentage of ceramic particles in the initial suspension. The Figure 2.7 gives an example of a ceramic part before and after firing (debinding and sintering), this part being fabricated with a ceramic suspensions charged at 65\% (CTTC, Limoges, France).

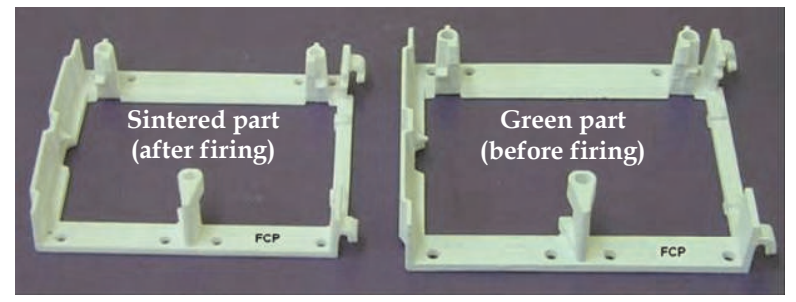

Fig. 2.7. Ceramic part made by ceramic stereolithography before and after firing; courtesy of the CTTC (Limoges, France)

Low percentage of ceramic particles (less than 50\%) leads to a shrinkage of typically more than $25 \%$. In such configurations, the sintered part shows high deformations if not cracks.

As a consequence every suspension will lead to a specific shrinkage. This value has to be precisely known in order to fabricate a bigger green part and to have a sintered part at the right size and shape. This initial oversizing is a very delicate step because the shrinkage, for complex geometries, may not be isotropic. The shrinkage is however identical from one fabrication to another.

\subsection{Conclusion}

Every element in the ceramic suspension used for the fabrication by ceramic stereolithography is very important because it sets all the future properties of the final fired ceramic part. Regarding the mechanical strength of the green part during the fabrication process itself, the fabrication definition, the final part density, its shrinkage during debinding and sintering, ..., every suspension has to be very precisely studied in order to obtain a successful compromise between all these parameters.

One major key is to go to high volume percentage of ceramic particles in the suspension because this will lead to highly dense ceramic part (high strength) and low shrinkage during the firing steps (low deformation). The drawbacks of this high percentage are a loss in lateral definition mainly because of the light scattering phenomena due to the presence of the ceramic particles. 
However by refining enough all these parameters and with a small diameter laser, a manufacturing accuracy close to $50 \mu \mathrm{m}$ can be reached. Combining this accuracy and the ability to manufacture dense ceramic parts with a very complex geometry, this technology gives a very high level of freedom for the design of high performances millimetre wave components.

\section{Advanced components for millimetre wave applications}

In this section, we now focus on advanced RF components fabricated by stereolithography process. Theoretical and experimental structures are compared for validating our approach.

\subsection{D ceramic woodpile crystal}

One of the most complex devices we fabricated is a ceramic woodpile (layer-by-layer) crystal. Such device is representative of the capabilities of the 3D stereolithography process. We describe two test structures based on the woodpile crystal.

The first one is a waveguide located in the woodpile (Delhote et al., 2007a). The geometrical sizes, shape and location of the waveguide into the ceramic woodpile have been optimized in order to maximize its bandwidth and the matching between this guide and the Input/Ouput feeding WR waveguides. The 3D Electromagnetic Band Gap (EBG) crystal has been designed, optimized and manufactured in one monolithic piece with Zirconia $(\varepsilon r=31.2$ at $30 \mathrm{GHz}$ ) by the 3D ceramic stereolithography process. It experimentally exhibits a very large bandgap superior to $30 \%$ and the waveguide located in such woodpile provides a measured $20 \%$ bandwidth around $26 \mathrm{GHz}$ while keeping a return loss inferior to $-10 \mathrm{~dB}$. This work focused on the improvement of the electrical performances of the waveguide by enhancing two major points. First, a close attention was paid to maximize the matching between an input standard WR waveguide and the EBG one without any taper by optimizing the configuration of the default. Then, the waveguide bandwidth has been improved simply by enlarging the EBG material complete bandgap. This last purpose has been reached by having recourse to high permittivity ceramic for the manufacturing and optimizing the filling factor, height and width of the woodpile's rods. Of course these different parameters have to agree the constraints imposed by the waveguide geometry. The lattice constant of the woodpile in the horizontal $(\mathrm{x} 0 \mathrm{y})$ plane is refereed as $a$, the rod width $w(=a / 4)$, the rod height $h$. Considering the chosen configuration, the $h / w$ ratio has to be equal to 0.9 and the filling factor, representing the percentage of dielectric in a unit cell, equal to $25 \%$. The chosen dielectric is the Zirconia ceramic known to present a permittivity of 31.2 at 30GHz. Such EBG material provides a 33\% complete bandgap around the normalized frequency $a f / c$ ( $f$ being the central frequency of the complete bandgap $(\mathrm{Hz})$ and $c$ the speed of light in vacuum) of 0.474 . By taking into account the manufacturing tolerances of the $3 \mathrm{D}$ ceramic stereolithography, the $w$ parameter has been chosen equal to $790 \mu \mathrm{m}$, making the complete bandgap appearing between 25 and $35 \mathrm{GHz}$ in the Ka band $(26-40 \mathrm{GHz})$. All the dimensions of the EBG crystal were first defined by computing its band diagram and its first Brillouin zone (see Figure 3.1) applying the plane wave method. Then, the dimensions were optimized applying 3D electromagnetic simulations based on a Finite Element Method (FEM)

Fig.3.2 (a) presents the fabricated woodpile. This structure was fabricated in only one monolithic piece. In order to measure its transmission (S21) and reflexion (S11) parameters, it was inserted in a measurement support as shown in Figure 3.2 (b). 

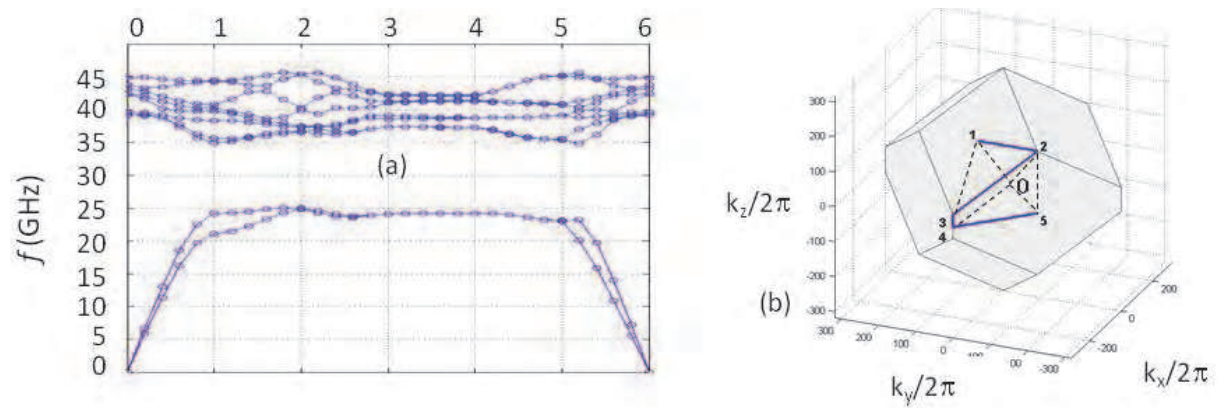

Fig. 3.1. (a) Band diagram of the proposed photonic crystal. (b) First Brillouin zone: the 6 high symmetry points used for the calculation are also displayed.
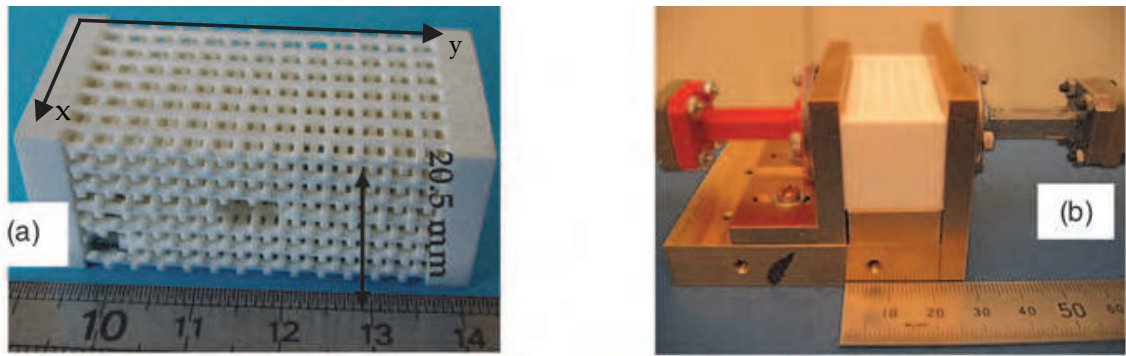

Fig. 3.2. (a) Monolithic waveguide located in a woodpile manufactured with Zirconia by ceramic stereolithography. (b) Woodpile waveguide inserted in its support feeded by two WR28 waveguides.

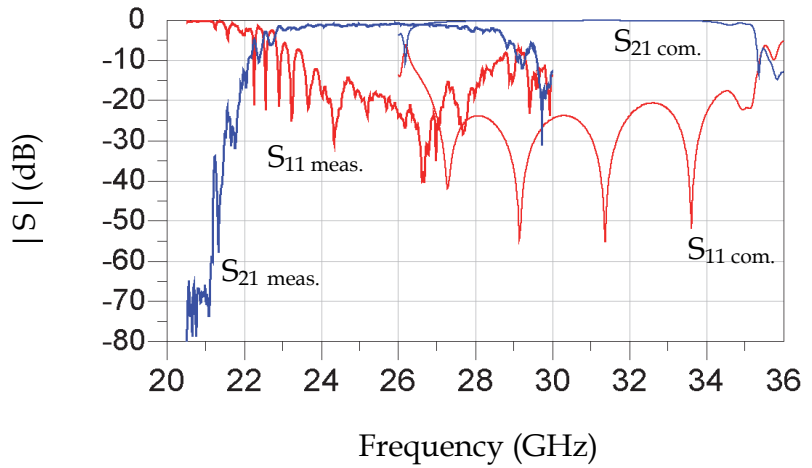

Fig. 3.3. Monolithic waveguide located in a woodpile manufactured with Zirconia by ceramic stereolithography. Experimental (meas.) and computed (com.) scattering parameters.

Figure 3.3 compares the theoretical and experimental scattering parameters of the waveguide. The waveguide band pass appears to be theoretically equal to $29.4 \%$ around 
$30.85 \mathrm{GHz}$ while keeping a return loss inferior to $-10 \mathrm{~dB}$. This waveguide takes advantage of almost the entire complete band gap provided by the woodpile. As presented in Figure 3.3, the experimental waveguide bandpass appeared to be centered around $26 \mathrm{GHz}$ instead of $30.85 \mathrm{GHz}$. This shift is due to fabricating discrepancies. Some misshapes have occurred, making the $y$ axis and $x$ axis rods respectively 800 and $500 \mu \mathrm{m}$ high instead of $710 \mu \mathrm{m}$. The rod width is also closer to $800 \mu \mathrm{m}$. However the experimental structure presents an interesting $19.6 \%$ bandpass around $26 \mathrm{GHz}$ for a return loss inferior to $-10 \mathrm{~dB}$. The insertion losses appear very acceptable with the measured values from 0.8 to $1.7 \mathrm{~dB}$ in the band pass. The second example concerns a 3D crystal millimetre resonant cavity (Delhote et al., 2007b). Electromagnetic properties of the previous ceramic woodpile structure are exploited in order to design an air resonant cavity. For creating such a cavity, we consider a defect inside the woodpile. The unloaded quality factor of the resonator depends on the dielectric material performances and on the crystal efficiency for limiting as much as possible the leakage through the crystal periods. The cavity dimensions obviously fix its working frequency and have to be precisely taken into account in order to adjust the chosen frequency inside the crystal frequency band gap. As said previously, the leakage can greatly reduce the unloaded $Q$. It can only be limited by adding a large number of crystal periods surrounding the cavity but this choice also heavily increases its volume as compared to the cavity size. Moreover, this larger number of period increases the losses provided by the dielectric material. Shielding the whole structure with metal allows for efficiently eliminating the leakage issue but can reduce the unloaded $Q$ by adding extra losses due to the metal itself. It has also to be considered that the closer the metal is to the air cavity, the higher the metallic losses are. However the radiative are very high and high unloaded quality factor can not be reached without a metal shield surrounding the whole structure. We investigated the best compromise between the size of a shielded 3D crystal and the unloaded quality factor value in order to manufacture and test such cavity. Two types of test structures were fabricated with success using zirconia and alumina. A specific low loss alumina has been developed with our partners. It presents a very challenging loss tangent of 5 10-5. Figure 3.4 describes the zirconia crystal. The computed E field distribution at the resonant frequency is described. As we can see, it is well confined in the air cavity.

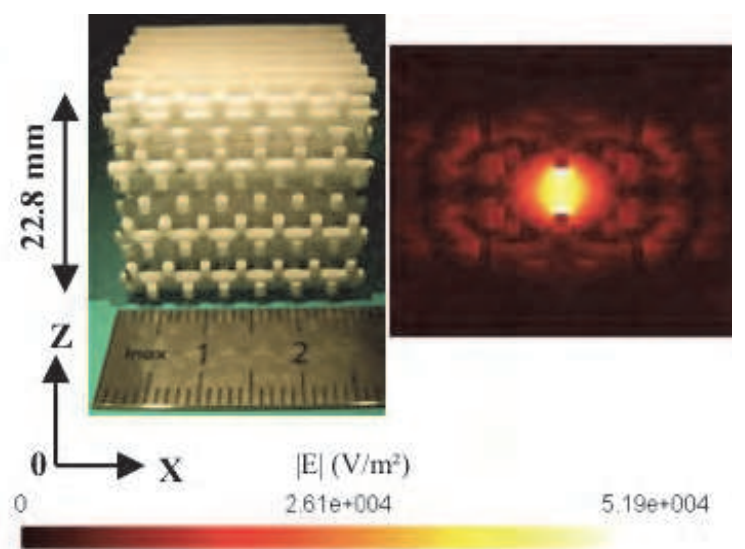

Fig. 3.4. Manufactured Zirconia layer-by-layer crystal. $|\mathrm{E}|$ field at $\mathrm{f}_{0}$ 
Figure 3.5 (a) displays the measured transmission parameter for the zirconia crystal. The working frequency is $31.5 \mathrm{GHz}$ and the unloaded quality factor is 990 , which is very close to the value predicted by computations. Figure 3.5 (b) presents the measured transmission parameter for the alumina crystal air resonant cavity. The measured unloaded $Q$ is about 4000. Due to the very low loss tangent, a theoretical value of the unloaded Q equal to 30000 could be reached. But for measuring such value, the experimental set up needs to be improved.

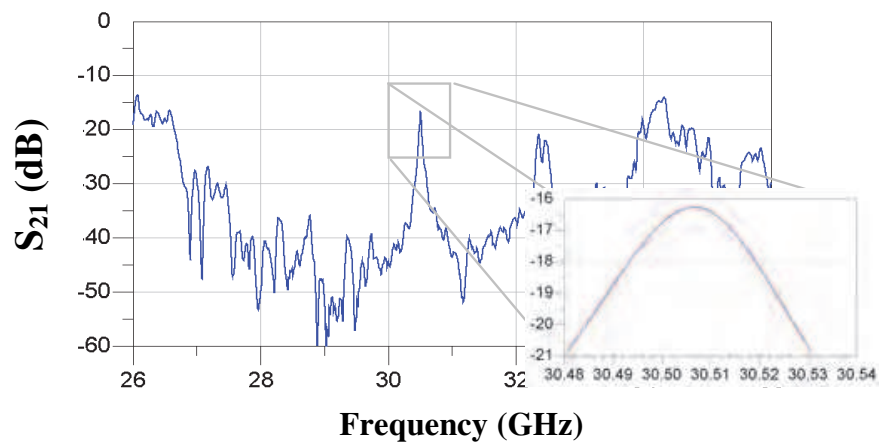

(a)

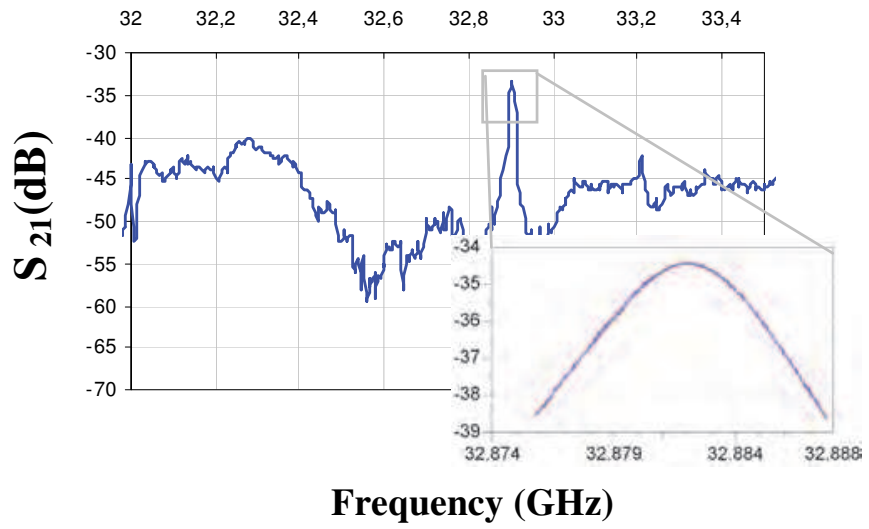

(b)

Fig. 3.5. (a) Measured transmission parameter for the zirconia 3D crystal air cavity. Close view around the working frequency. (b) Measured transmission parameter for the alumina $3 \mathrm{D}$ crystal air cavity. Close view around the working frequency

\subsection{Alumina lens antennas}

In collaboration with IETR UMR CNRS 6164, University of Rennes 1, Rennes, France, we designed, fabricated and characterized integrated lens antennas made in Alumina (Nguyen et al., 2010). They are built through ceramic stereolithography. The operating frequency is in the $60-\mathrm{GHz}$ band. Linear corrugations are integrated on the lens surface to reduce the effects 
of multiple internal reflections and improve the antenna performance. The antenna geometry is described in Figure 3.6. It consists of a synthesized elliptical lens made in Alumina; the dielectric characteristics of Alumina have been measured at $10 \mathrm{GHz}$ using a resonant cavity: $\varepsilon_{r}=9.0, \tan \delta=5 \times 10^{-5}$. The same values are chosen at $60 \mathrm{GHz}$. The antenna is excited by a rectangular waveguide with an integrated impedance matching taper. Three antenna prototypes have been fabricated using the stereolithography process. The first one (ILA\#1, Fig. 6a) and second one (ILA ${ }_{\# 2}$ ) have corrugations of different size: their widths equal $300 \mu \mathrm{m}$ for ILA\#1 and $400 \mu \mathrm{m}$ for ILA I $_{\# 2}$. The other corrugation dimensions depth and spacing equal $700 \mu \mathrm{m}$ and $1200 \mu \mathrm{m}$ respectively. The third lens antenna (ILA\#3) has a smooth surface, i.e. no corrugation. Considering two different corrugation widths and depths allows assessing the fabrication limitations in terms of resolution and minimum size of small 3-D objects, whereas comparing ILA configurations with and without corrugations enables one to highlight the impact of these corrugations upon the antenna performance. Here all lenses have the same total diameter and height, and are fed by the same impedance matching taper.
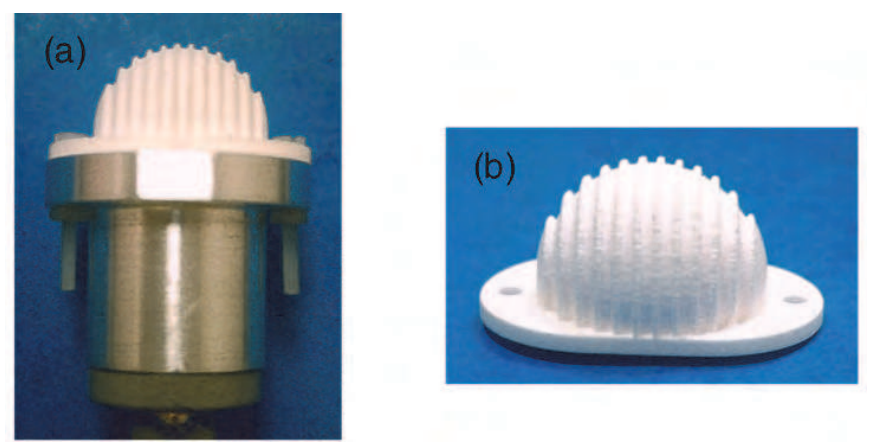

Fig. 3.6. Synthesized elliptical ILA (ILA\#1 and 2) fabricated by ceramic stereolithography. (a) Antenna prototype (after assembly). (b) Cross-section view of the Alumina lens alone.

The measured reflection coefficients of the three lens antennas are smaller than $-10 \mathrm{~dB}$ from $55 \mathrm{GHz}$ to $65 \mathrm{GHz}$. The radiation patterns measured at $60.5 \mathrm{GHz}$ are represented in Figure 3.7 They are in good agreement with the FDTD simulations. The slight asymmetry observed on the measured beams probably comes from lens deformation. Additional experimental results have confirmed that these patterns are very stable between $55 \mathrm{GHz}$ and $65 \mathrm{GHz}$.

The antenna gains are plotted in Figure 3.8. They have been measured with the comparison method using a 20-dBi standard gain horn in $V$-band. As expected this figure confirms that the gain variations versus frequency are smaller when using fine corrugations $\left(\mathrm{ILA}_{\# 1}\right)$. At $60 \mathrm{GHz}$ the gain and directivity of ILA $\mathrm{\# 1}_{1}$ equal $19 \mathrm{dBi}$ and $19.9 \mathrm{dBi}$, respectively.

This study demonstrates that the stereolithography process can be used to fabricate specific antenna lens with high performances. Measurements have shown that the antenna performances are very stable over the $55-65 \mathrm{GHz}$ band. The agreement between the experimental and numerical results is very satisfactory despite some fabrication issues (mechanical stress observed along the dielectric tapers and lens bases). In particular, for 19$\mathrm{dBi}$ gain antennas, the total amount of loss at the center frequency $(60.5 \mathrm{GHz})$ is lower than $0.9 \mathrm{~dB}$, corresponding to a radiation efficiency of $80 \%$. 


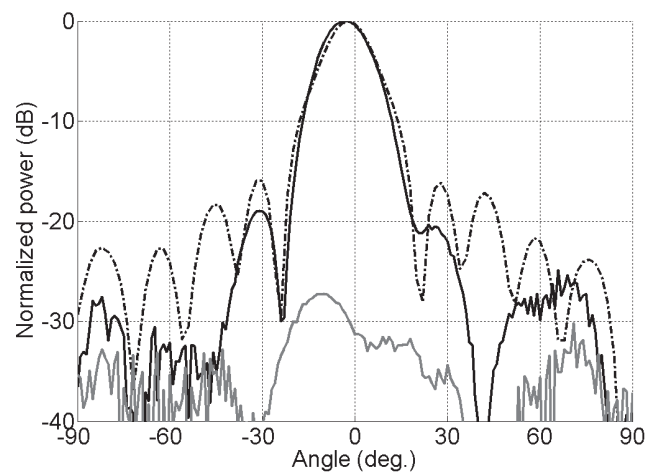

(a)

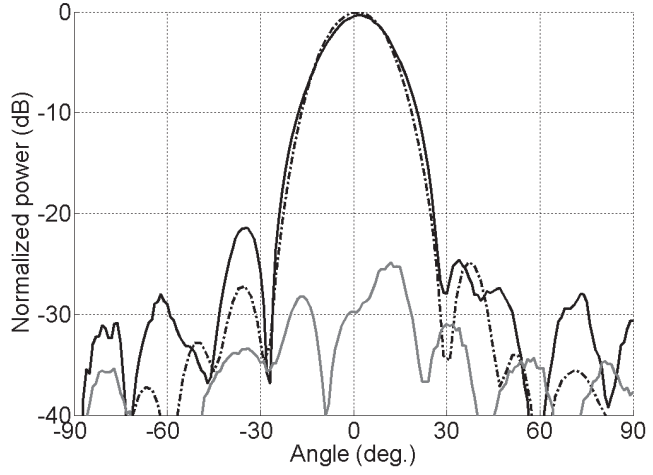

(b)

Fig. 3.7. Radiation patterns of ILA\#1 at $60.5 \mathrm{GHz}$. (a) E-plane. (b) H-plane. —: Measured copolarization component. ---: Computed co-polarization component. —: Measured crosspolarization component.

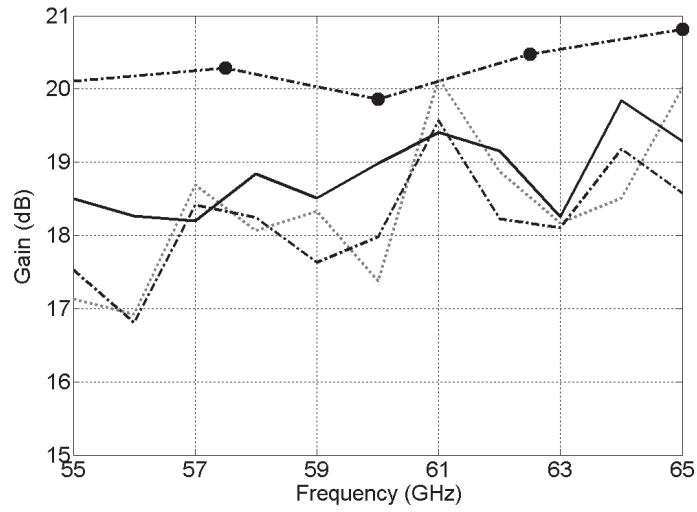

Fig. 3.8. Measured gain of the three lens antennas. —: ILA\#1 (300 $\mu$ m-thick corrugations). ---: ILA\#2 (400 $\mu \mathrm{m}$-thick corrugations). ....: ILA\#3 (no corrugation). The line with symbols (---) represents the theoretical directivity of ILA\#1. 


\subsection{Ceramic bandpass filters}

\subsubsection{Bandpass filter based on periodic structure}

Several bandpass filters have been designed and fabricated applying the stereolithography process. The first example concerns the design of a high unloaded $Q$ factor $\left(Q_{u}\right.$ factor $)$ bandpass filter working in the Ka band (Delhote et al., 2007c). Such filter is based on periodic structures and made of Zirconia and high performance $\mathrm{Ba}_{3} \mathrm{ZnTa}_{2} \mathrm{O}_{9}$ (BZT) ceramics. To our knowledge, BZT is mainly used for the manufacturing of simple cylinder-shaped resonators by standard processes such as pressing or molding. It is the first time 3D ceramic stereolithography has been used to manufacture complex innovative filtering devices based on periodic arrangements with high performance ceramics. Figure 3.9 describes the filter under test. It consists of a resonant air cavity surrounding by Bragg reflector as shown in Figure 3.9 (a). Along the $\mathrm{x}$ axis, four period reflectors are placed to strongly contain the field in the cavity. The fourth exterior wall of these four period reflectors is $3 \lambda \mathrm{g} / 4$ thick to provide an extra mechanical robustness for this wall which remains in free space.

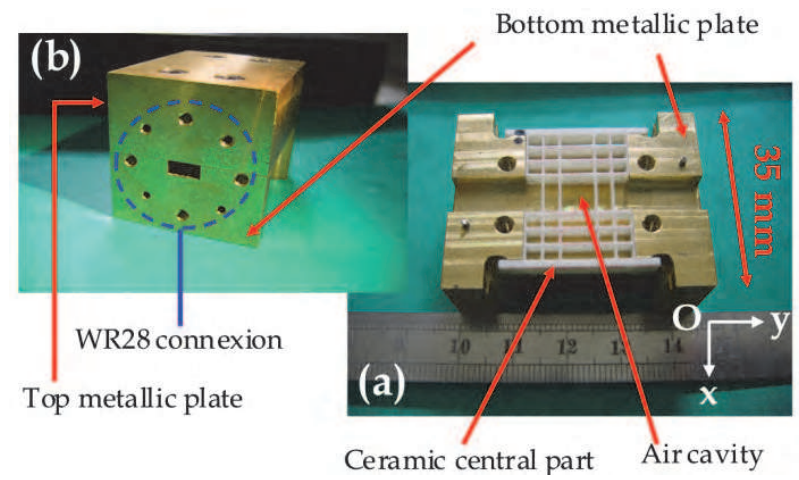

Fig. 3.9. (a) Manufactured single cavity central ceramic part on its brass metallic support (bottom metal plate). (b) The front/rear view of the whole structure is shown in the upper left corner

The air cavity is excited by standard WR28 waveguide placed on each side of the central part along the y axis, and only two period reflectors are placed along this axis to excite the resonant mode of the cavity. The other parts of the structure are metallic plates put on the bottom and on the top (Figure 3.9(b)) of the ceramic central part. The whole structure can be easily connected to standard WR 28 waveguides on its front and rear faces for its measurement (see Figure 3.9(b)). The main interest of this structure is that its performances not only depend on the metallic losses but also on the dielectric ones, making it possible to reach high unloaded quality factor with the proper dielectric material.

During this study, we considered two ceramic: Zirconia with a relative permittivity of 31.2 and a loss tangent $(\tan \delta)$ of $1.810^{-3}$ at $30 \mathrm{GHz}$, and the Temex Ceramics $\odot$ BZT with a relative permittivity of 30.2 and a loss tangent of $\mathrm{f}(\mathrm{GHz}) / 173600$, that means a loss tangent of $1.910^{-4}$ at $33 \mathrm{GHz}$. In order to demonstrate the proof of concept of the device described in Figure 3.9, a test structure was fabricated using zirconia. The fabricated dimensions are as follow: the cavity is $7 \mathrm{~mm}$ by $5.86 \mathrm{~mm}$ by $3.5 \mathrm{~mm}$. Dielectric walls are $0.47 \mathrm{~mm}$ thick and separated by $2.45 \mathrm{~mm}$, making the average manufacturing accuracy close to $50 \mu \mathrm{m}$. The outside dimensions are $27.2 \mathrm{~mm}$ by $26.4 \mathrm{~mm}$ by $3.5 \mathrm{~mm}$. It represents a $0.95 \%$ manufacturing 
discrepancy compared to the theoretical values. This structure was specifically designed for measuring the $Q_{\mathrm{u}}$ factor. The structure, measured with a Hewlett Packard 85107A network analyzer, exhibits a $\mathrm{f}_{0}$ working frequency of $33.564 \mathrm{GHz}(1.7 \%$ shift compared to the theoretical value of $32.99 \mathrm{GHz})$ and a $-3 \mathrm{~dB}$ bandwidth $\left(\Delta \mathrm{f}_{-3 \mathrm{~dB}}\right)$ of $42 \mathrm{MHz}$. The scattering parameters at $33.564 \mathrm{GHz}$ are measured as follow: $S_{21}=-6.48 \mathrm{~dB}, S_{11}=-9.91 \mathrm{~dB}$ and $S_{22}=-$ $9.46 \mathrm{~dB}$. The unloaded quality factor appears to be about 2400 whereas the theoretical value is 2800 (see Figure 3.10) However this value is less than the $3000 \mathrm{Q}_{\mathrm{u}}$ given by a standard brass shielded air cavity having the same dimensions as the air cavity of the central ceramic part.

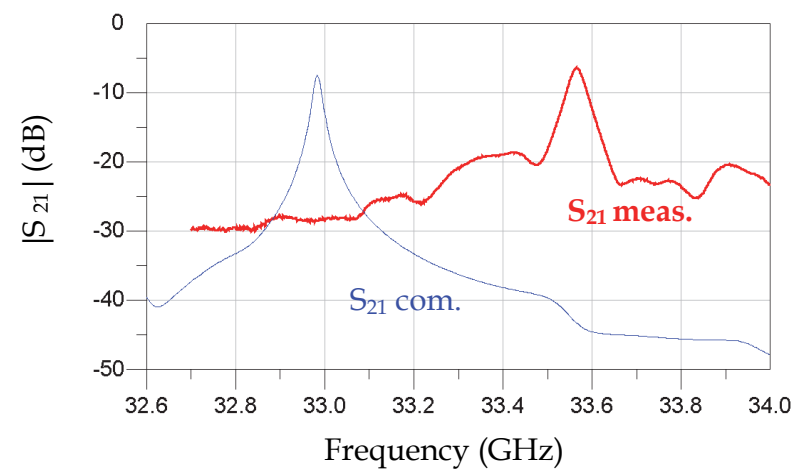

(a)

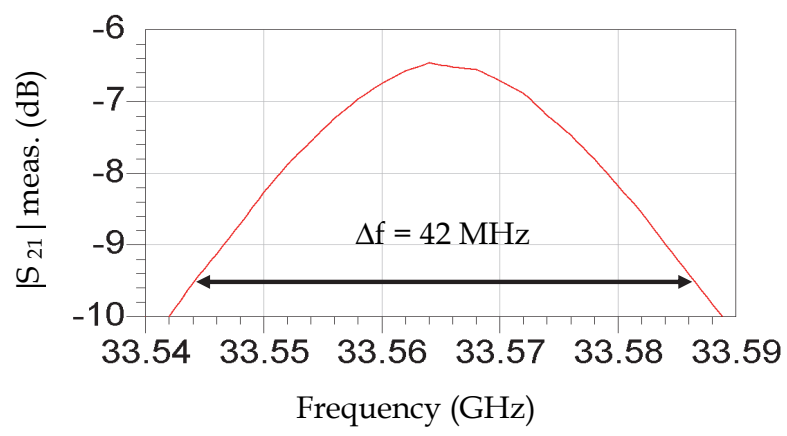

(b)

Fig. 3.10. (a) Computed (thin line) and measured (thick line) transmission parameter of the hybrid single cavity. (b) Close view of the measured (meas.) S21 parameter.

In order to increase the unloaded $\mathrm{Q}$ factor and consequently decrease the insertion losses, the Zirconia can be replaced by the BZT. In this case, the $Q_{u}$ factor is equal to 7000. Such value cannot be reached by a classical metallic cavity at this frequency. As shown in Figure 3.11, several pieces of BZT were fabricated by stereolithography with an average manufacturing accuracy close to $80 \mu \mathrm{m}$. New tests and fabrication procedure are currently under consideration in order to improve the fabrication accuracy with BZT. 


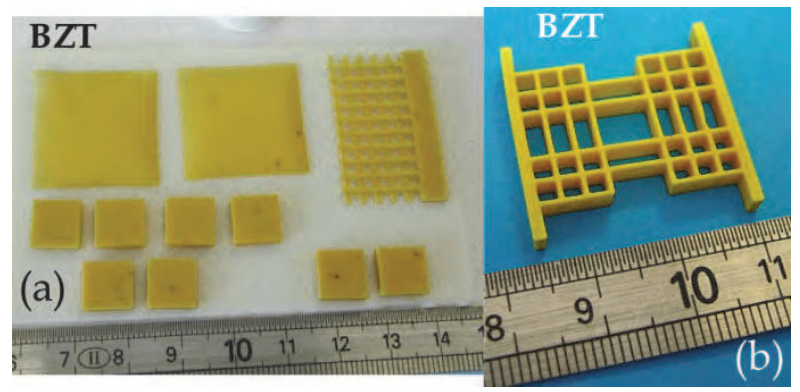

Fig. 3.11. Manufactured pieces out of BZT made to verify its compatibility with the ceramic stereolithography process. (a) Three different parts are presented: $100 \mu \mathrm{m}$ thick plates, parallelepipeds and complex structure composed of crossed bars. (b) Manufactured single cavity resonant structure out of BZT

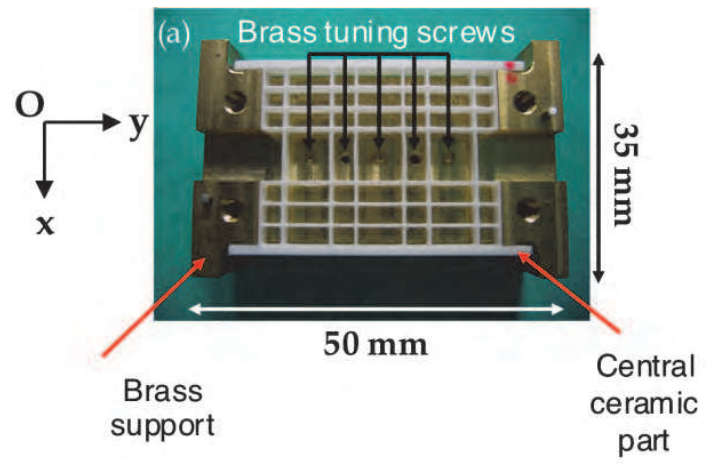

(a)

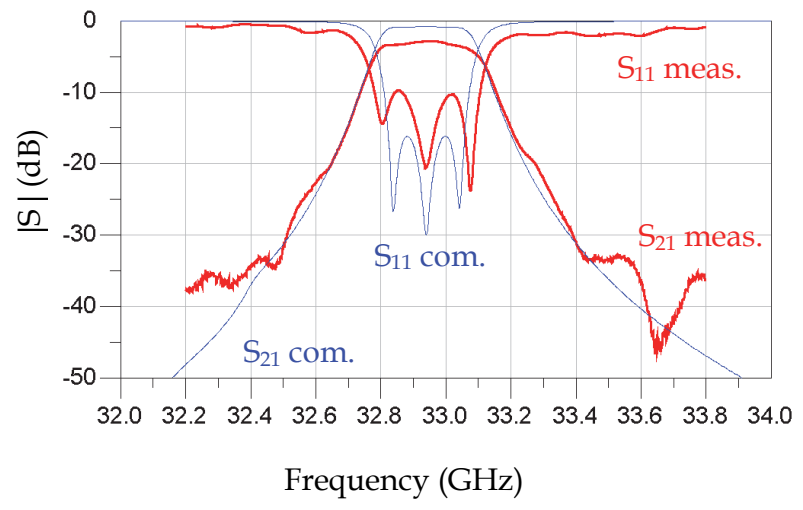

(b)

Fig. 3.12. (a) Manufactured three pole central ceramic part in its brass support (i.e. bottom metallic plate). (b) Measured (thick line) and theoretical (thin line) responses of the three pole filter. 
Finally, a narrow bandpass three pole filter was fabricated using Zirconia because of better manufacturing accuracy and its sufficient characteristics to provide a narrow bandwidth in the Ka band. The three cavities required for the three pole filter are placed along the $y$ axis. The coupling distance between them were dimensioned to provide about $1 \%$ bandwidth at $33 \mathrm{GHz}$ and a ripple of $0.1 \mathrm{~dB}$. So, cavities have the same dimensions as the previous ones and the spacing between the cavities is $2.85 \mathrm{~mm}$. The outside dimensions are $27.48 \mathrm{~mm}$ by $39.75 \mathrm{~mm}$ by $3.56 \mathrm{~mm}$. Brass tuning screws are placed above each cavity and each coupling spacing to refine the different coupling coefficient between the cavities. The three pole filter is described in Figure 3.12 (a) and its S parameters in Figure 3.12 (b). As we can see, experimental (after tuning) and theoretical behaviors are in satisfying agreement.

\subsubsection{Alumina resonator shielded in a alumina cavity}

The second example of resonant structure dedicated to RF filtering applications, concerns the fabrication of an alumina resonator shielded in a alumina cavity (Delhote et al., 2007d). This device is described in Figure 3.13. It is composed of two parts: a main structure containing the dielectric resonator (DR), its support and the surrounding cavity manufactured in one alumina piece, and a alumina plate placed on the top of the main part in order to close the cavity.
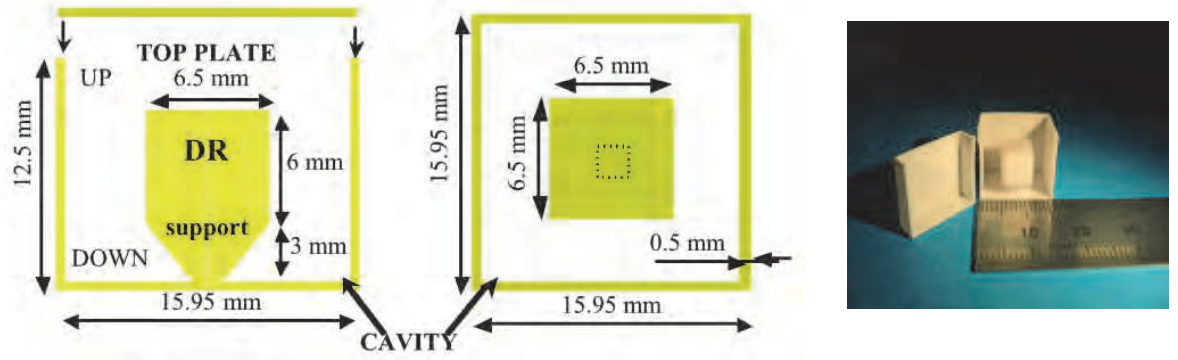

Fig. 3.13. Design of the alumina resonant structure with its main dimensions: inside side view, top view and fabricated structure

The dielectric resonator is parallelepiped because of its manufacturing simplicity and made out of low dielectric loss alumina. This ceramic has been characterized with a permittivity of 9.1 and a low dielectric los tangent of $1.310^{-4}$.The DR support is also designed taking into account technological constraints, and inversed pyramidal shape is chosen considering this criteria. The surrounding alumina walls are 500- $\mu$ m thick. This thickness permits to avoid spurious resonant modes inside the ceramic walls and to ensure overall mechanical robustness. As we mentioned, the resonator, its support and the cavity are manufactured in only one piece. During the process, this piece needs to remain open on its top in order to correctly clean the monomer surplus. Then, an alumina plate, having the same outside dimensions as the main part, is centered and glued on top of it in order to close the cavity. The DR resonant mode is the TM111 mode and the working frequency is fixed at $12.57 \mathrm{GHz}$. The DR dimensions are $6 \mathrm{~mm} \times 6 \mathrm{~mm} \times 6.5 \mathrm{~mm}$ and the cavity is $15.95 \mathrm{~mm} \times 15.95 \mathrm{~mm} \times$ $13.5 \mathrm{~mm}$. The DR support height is $3 \mathrm{~mm}$. The surrounding cavity is dimensioned to provide a good compromise between frequency isolation and high unloaded Q. Its external faces are metalized with Epotek E202 epoxy silver glue (conductivity close to $6.610^{5} \mathrm{~S} / \mathrm{m}$ ). 
This method has been chosen for its simplicity to quickly provide good metallic shielding. The top and bottom faces of the cavity are first polished with $10 \mu \mathrm{m}$ grit size diamond polishing discs and Heraeus KQ500 etchable gold is applied on them, in order to provide a $5 \mu \mathrm{m}$ thick layer in gold after baking. Input/output (I/O) RF coplanar excitation accesses are then etched on the top or bottom face of the structure. It is suitable to demonstrate the feasibility of such structure. Figure 3.14 (a) presents the final structure with the coplanar test probes. The experimental transmission parameter is presented in Fig.3.14 (b). It is compared to EM simulations. The two electrical behaviors are in very good agreement. That permits to validate both the numerical approach and the experimental process. The DR resonant frequency $\left(\mathrm{f}_{0}\right)$ is equal to $11.87 \mathrm{GHz}$ and unloaded $\mathrm{Q}$ factor is extracted by measuring the frequency bandwidth $\Delta \mathrm{f}$ at $-3 \mathrm{~dB}$ from the maximum transmission level $(-31 \mathrm{~dB})$. Then by considering this weak transmission level, the unloaded $Q$ factor is estimated by the usual formula $\mathrm{Q}_{\mathrm{u}}=\mathrm{f}_{0} / \Delta \mathrm{f}$. Its experimental value is close to 3900 as expected by numerical computation.

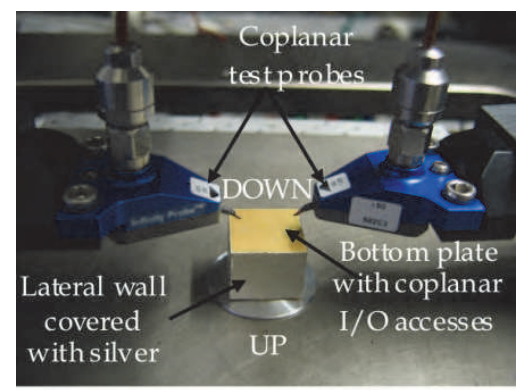

(a)

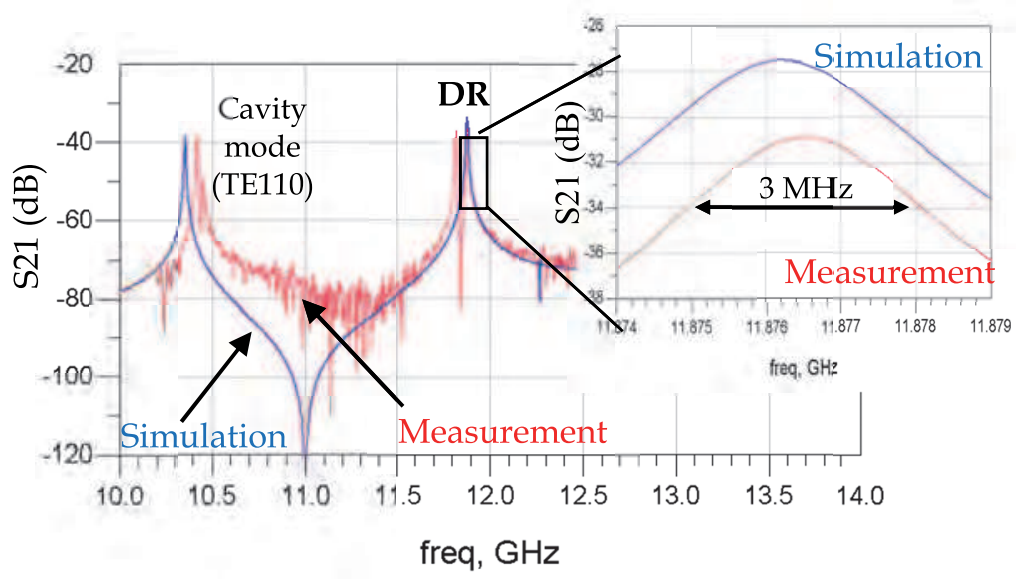

(b)

Fig. 3.14. (a) Final structure under test. The structure is upside down comparing to Fig.3.13 (a). (b) Measured and computed results of the structure: wide band and close view of the S21 parameter around DR resonant frequency at $11.876 \mathrm{GHz}$. 
High unloaded Q ( 3900) is reached with the presented shielded DR made out of alumina. Its compactness and its ability to be reported on a substrate carrier by standard means (flipchip or bumps) make this dielectric resonator very interesting for many applications. By providing better metallization (such as gold) instead of silver glue on the lateral walls, unloaded quality factor could theoretically raise to 7000 .

\subsubsection{3-D pyramidal and collective Ku bandpass alumina filters}

The last example described in this book chapter concerns a 3-D pyramidal and collective $\mathrm{Ku}$ bandpass filters made in Alumina by ceramic stereolithography (Khalil et al., 2011). The objectives of this work are to propose alternative technologies for the manufacturing of low insertion loss and compact (footprint less than $15 \mathrm{~mm}$ by $15 \mathrm{~mm}$ ) band pass filters in the upper $\mathrm{Ku}$ band $(17.5 \mathrm{GHz})$ for space applications. This filter has to remain flat (thickness less than $2.5 \mathrm{~mm}$ ) and need coplanar input/output accesses to be connected inside a communication module by gold wire bonding or with the flip-chip technology. Temperature stability is a plus and the Alumina has interesting properties in this field. Based on these considerations, the chosen technology for this purpose has been the 3D ceramic stereolithography because of its ability to fabricate 3D compact and low loss Alumina resonators. These resonators will also be shielded with high conductivity metals such as Gold, Silver or Copper. Finally, wet chemical etching or laser ablation techniques will be considered for the etching of the coplanar accesses in the shielding. Nine low footprint Alumina filters are manufactured in one single fabrication by a 3D ceramic stereolithography process. Thank to this technology, high unloaded quality factor resonator (above 1000) have been fabricated and associated to create a low footprint 4-pole Chebyshev filter. Its shielding has been created with a Copper and Gold sputtering technique and its input and output coplanar accesses have been etched by a laser ablation technique. The association of these 3D technologies for the collective manufacturing of many filters in one ceramic part, has led to a low insertion loss filter working around $17.5 \mathrm{GHz}$. Figure 3.15 displays the shape of the selected 4-pole filter as well as its main dimensions in millimeter.
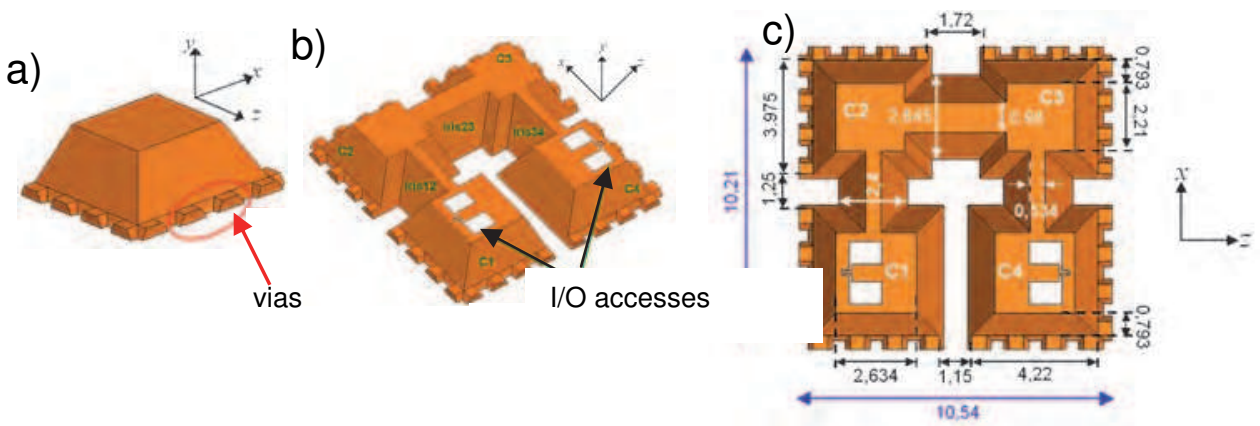

Fig. 3.15.(a) Pyramidal vias integrated at the bottom of the resonator. (b) Final 4-pole filter geometry integrating the pyramidal resonators and vias. (c) Main dimensions of the filter in millimeter $(\mathrm{mm})$. 
Figure 3.16 presents the ceramic part before and after metallization and etching of the coplanar accesses. For cost reason, the sputtering of Copper instead of Gold has been preferred. However a Gold coating of $1 \mu \mathrm{m}$ has been put on top of the $5 \mu \mathrm{m}$ thick Copper layer to avoid the oxidization of this layer. The etching step has been performed with a 1080nm YAG laser ablation system. This technology has been chosen because of its sufficient accuracy, its capability of removing all ceramic particles on its path and because of its ability to work with 3D parts.
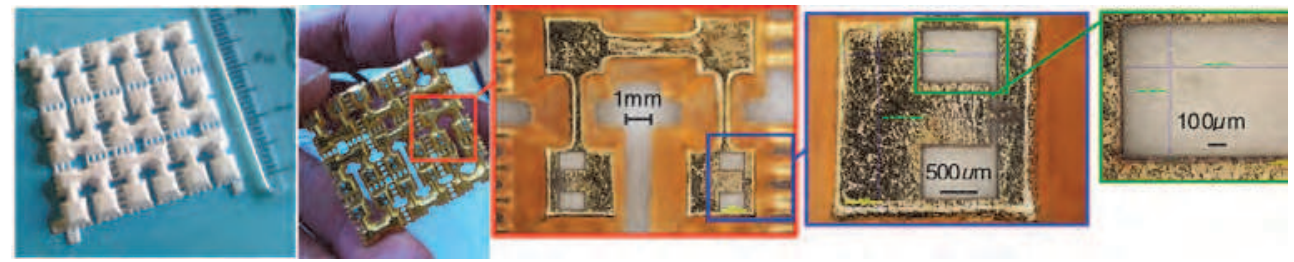

Fig. 3.16. Different view of the filters before and after their metallization with Copper and Gold and their etching with a YAG laser.

Figure 3.16 also shows a close view of the etched accesses. The dimensions of these patterns have been very accurately drawn with a measured accuracy of less than $+/-5 \mu \mathrm{m}$. The nine filters have been measured with $50 \mathrm{Ohms}$ coplanar probes on an HP vectorial network analyzer. The experimental S parameters of a typical response are displayed on Figure 3.17 as well as the filtering specifications.

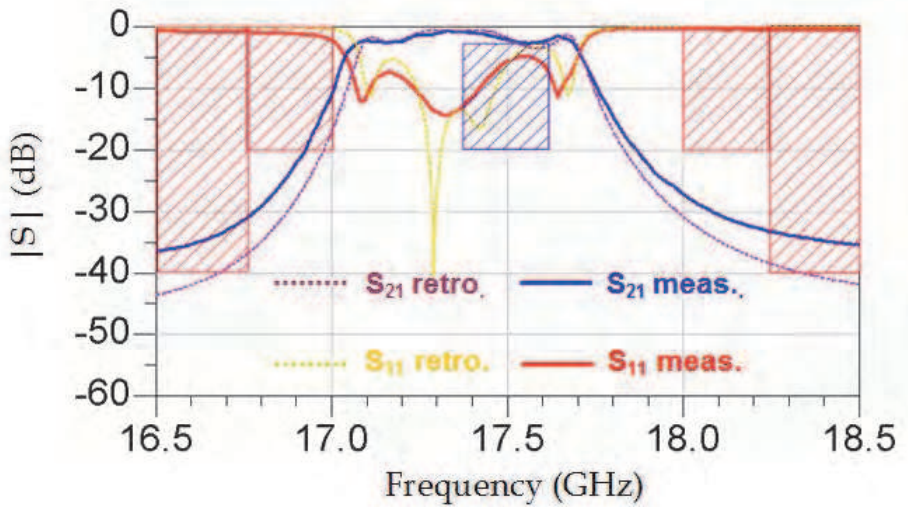

Fig. 3.17. Measured S parameters (solid line) and retro-computations (dashed line).

The experimental results are a central frequency of $17.36 \mathrm{GHz}$ and an equi-ripple band pass of $560 \mathrm{MHz}$. The insertion loss is $0.7 \mathrm{~dB}$, showing that the $\mathrm{Q}$ factor of each resonator is indeed close to 1000 but the in band ripple is about $2 \mathrm{~dB}$. The return loss is only $5 \mathrm{~dB}$ and the rejection is not sufficient, especially in the lower out of band frequency region. These values coming from a very first fabrication are not satisfying. In order to determine the causes, the manufactured filters main dimensions have been measured. It has appeared that most dimensions present a $+/-100 \mu \mathrm{m}$ variation from the theoretical values. Retro-computations have been performed with these dimensions and, as seen on the Figure 3.17, the 
experimental behavior has been fully explained. It appears that the inaccurate irises between cavities are mainly responsible of the degraded frequency behavior. Another refined manufacturing of the ceramic part is already ongoing, taking into account the experimental data extracted from this trial. The insertion loss are however acceptable for the filtering specifications (less than the required $3 \mathrm{~dB}$ ) and the next refinements will be mainly dedicated on obtaining the right pass band, return loss and frequency isolation. The design itself, the overall part geometry, metallization and etching steps have shown that they are compliant with the objective of a small footprint and low insertion loss filter dedicated to space applications. Many 3D technological challenges have thus already been addressed. The proof of concept has been demonstrated.

\section{Shape optimization methods}

The last section focuses on advanced design of microwave and millimeter-wave components applying shape optimization techniques. These techniques are utilized for optimizing the shape of dielectric components and optimized structures are fabricated using ceramic 3D stereolithography process.

\subsection{Shape optimization in the context of electromagnetic problems}

Shape optimization methods reside in determining the optimal shape of an object in order to satisfy given specifications. Several approaches have been developed in the context of computer aided-design (CAD), particularly for solving mechanical problems (Sokolowski \& Zochowski, 1999; Allaire \& Jouve, 2002; Suri et al., 2002), and some of them can be adapted to electromagnetic (EM) problems (Mader et al., 2001; Kozak \& Gwarek, 1998; Byun et al., 2004). Considering an initial object embedded in a more global domain, shape optimization strategies can be classified into two categories: boundary optimization, which modifies the contour of the object; and topology optimization, which introduces local perturbations within the domain.

Compared to the classical parameter optimization strategy, which transforms the object with respect to its geometrical dimensions, shape optimization strategies allow accessing to a wider variety of shapes, i.e. of solutions, for the object to be optimized.

In the context of electromagnetic computer-aided design, numerical methods based on finite-elements or finite-differences have been extensively developed in the microwave engineer's community. Among boundary optimization techniques, the level-set (LS) method appears well-suited for solving electromagnetic problems (Kim et al., 2009), particularly with such discretized models; while on the other hand, the topology gradient (TG) method can be easily implemented as a topology optimizer for EM problems (Mader et al., 2001).

In both case, optimization of the numerical model is achieved thanks to a gradient evaluation calculated for a cost function related to the model behavior. The optimization strategy consists then in modifying the boundary or the topological elements iteratively in order to minimize the cost function.

Such numerical problems are generally constrained ones, which require the resolution of an adjoint problem, similarly to a geometrical design sensitivity approach (Akel \& Webb, 2000). However, considering shape optimization methods, the essential difference lies in the number of variables, which is often huge.

The level-set method (Allaire et al., 2003) is known for modeling propagating fronts and is based on the shape derivative, moving the boundary along the gradient direction during the optimization process. LS methods have been applied very successfully in many areas of scientific modeling and optimization. 
The topology gradient method evaluates a gradient concerning a small modification of the domain, translated into adding or removing material very locally. TG optimization was originally developed for mechanics design problems, and it has been recently adapted to other types of design problems, such as electrical and electronic devices.

The topology gradient and level-set methods coupled with a finite element method for optimizing microwave components have been demonstrated in 2-D (Assadi-Haghi et al., 2006) for optimizing the distribution of metal upon the surface of microstrip components and in 3-D (Khalil et al., 2008, 2009, 2010, 2011) for optimizing the distribution of a dielectric material within waveguide components.

\subsection{Topology-gradient (TG) and level-set (LS) methods coupled with finite elements 4.2.1 Finite element method (F.E.M)}

For analyzing a microwave component with a numerical method such as finite elements, the electromagnetic model is discretized into small elements before solving Maxwell's equations. Applying the finite element method, the following matrix equation is solved:

$$
A(\eta) E(\eta)=B
$$

where $A$ is a square symmetric matrix (Maxwell's operator) representing the geometrical and material properties of the discretized model, $B$ is the column vector of imposed sources, and $E$ the unknown field vector, solution of equation (4.1). Both $A$ and $E$ depend on $\eta$, a parameter characterizing the shape.

\subsubsection{Topology gradient (TG) method}

The topology gradient (TG) optimization procedure starts from an initial configuration of the domain and converges iteratively to the optimum shape using the gradient calculation. The later gradient is calculated at the first order by analyzing the sensitivity of the cost function with respect to a small perturbation of the domain $\Omega$.

We consider $\eta$ as the reference perturbation centered at origin. The perturbation centered at point $x$ and of size $h$, is characterized by:

$$
\eta_{x, h}=x+h \eta
$$

For calculating the gradient, the principle of topological asymptotic is utilized. The approach gives then a more precise expression of this gradient.

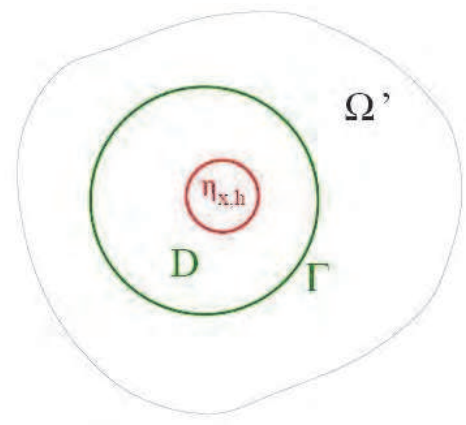

Fig. 4.1. Perturbation in the fixed domain D. 
The main idea is to isolate the perturbation in the fixed domain $D$ (Figure 4.1). The interest of this approach is to reduce the domain of problem to $\Omega^{\prime}=\Omega \backslash D$. The perturbation becomes a surface condition on the contour $\Gamma$. $A^{0}$ is the initial Maxwell's operator (without perturbation) and $A^{x, h}$ the perturbed operator. A similar notation is utilized for the electric field $E$.

The perturbation does not depend on the imposed sources, so the problem in (4.1) can be divided into two parts:

$$
\begin{aligned}
& A_{\Omega}^{0} E_{\Omega}^{x, h}=B^{0}-B_{\Gamma}^{x, h} \\
& A_{D}^{x, h} E_{D}^{x, h}=B_{\Gamma}^{x, h}
\end{aligned}
$$

assuming on contour $\Gamma$ that:

$$
E_{D}^{x, h} / \Gamma=E_{\Omega^{\prime} / \Gamma}^{x, h}
$$

When the perturbation is negligible $(h \rightarrow 0)$, the perturbed field on contour $\Gamma$ equals the initial field (without perturbation):

$$
E_{\Omega^{\prime} / \Gamma}^{x, h}=E_{\Omega^{\prime} / \Gamma}^{0}
$$

A classical approach for solving this class of problems is to minimize the cost function $J$ applying the Lagrange method where $L$, the Lagrangian function, is defined by:

$$
L\left(Y, \eta_{x, h}, P\right)=J\left(E^{x, h}\right)+\operatorname{Re} \operatorname{Tr}\left(P^{*}\left(A^{x, h} Y-B\right)\right)
$$

In our case, $Y$ is replaced by $E$, solution of (1):

$$
J\left(E^{x, h}\right)=L\left(E, \eta_{x, h}, P\right), \quad \forall P
$$

$P$ is the vector of Lagrange multipliers, an independent parameter that converts the constrained problem into an unconstrained one. The topology gradient is calculated by the difference between the perturbed cost function and the initial cost function (without perturbation):

$$
g_{x, h}=J\left(E^{x, h}\right)-J\left(E^{0}\right)+\operatorname{Re} \operatorname{Tr}\left(P^{*}\left(A^{x, h} E^{x, h}-A^{0} E^{0}\right)\right)
$$

If $J$ is a linear function of field $E$, one can write:

$$
\begin{aligned}
& g_{x, h}=\operatorname{Re}\left(\left(\partial_{E} J\left(E^{0}\right)\right)^{T}+P^{*} A^{0}\right)\left(E^{x, h}-E^{0}\right) \\
& +\operatorname{Re} \operatorname{Tr}\left(P^{*}\left(A^{x, h}-A^{0}\right) E^{x, h}\right)
\end{aligned}
$$

$P$ is then the solution of an adjoint problem: 


$$
\left(A^{0}\right)^{*} P=-\partial_{E} J^{*}\left(E^{0}\right)
$$

The topology gradient can be finally calculated as follows:

$$
g_{x, h}=\operatorname{Re} \operatorname{Tr}\left(P^{*}\left(A_{D}^{x, h}-A_{D}^{0}\right) E_{D}^{x, h}\right)
$$

One can note that, in the previous equations, superscripts $T$ and * denote respectively the transposition and conjugation operators.

After solving the direct and adjoint problems successively, the topology gradient is calculated for each element in the fixed domain $D$, then all topological elements with a negative gradient can be modified to minimize the cost function, and the topology gradient can be then evaluated with the new configuration. The procedure is repeated iteratively until a local minimum is attained.

\subsubsection{Level-set (LS) method}

The variables are defined on the contour of the object $\Omega$. It suffices to mesh $\Omega$ and to deform the contour $\eta$ according to the descent direction $\theta$. Let a bounded domain $D \subset \mathbb{E d}(\mathrm{d}=2$ or 3$)$ be the working domain in which all admissible shapes $\Omega$ are included, $\Omega \subset D$. In numerical practice, the domain $D$ will be uniformly meshed once and for all. The boundary of $\Omega$ is parameterized by means of a level-set function, following the idea of Osher and Sathian (Osher \& Sethian, 1988; Osher \& Santosa, 2001; Sethian \& Wiegmann, 2000). This level-set function $\psi$ is defined in $D$ such that:

$$
\left\{\begin{array}{lll}
\psi(x)=0 & \Leftrightarrow & x \in \partial \Omega \cup D, \\
\psi(x)<0 & \Leftrightarrow & x \in \Omega, \\
\psi(x)>0 & \Leftrightarrow & x \in(D \backslash \bar{\Omega}) .
\end{array}\right.
$$

In order to minimize $J$, a shape derivative is computed so that:

$$
J^{\prime}(\Omega)(\theta)=\int_{\partial \Omega} f(E, P, \eta) \theta . n d s
$$

where $f(E, P, \eta)$ is a scalar function, which depends on $E, P$ and $\eta$.

Therefore, we can define a descent direction in the whole domain $D$ by:

$$
\theta=-f . n
$$

where $n$ is the normal to the shape $\Omega$.

The evolution of the LS function is governed by the following Hamilton-Jacobi transport equation:

$$
\frac{\partial \psi}{\partial t}-f|\nabla \psi|=0
$$


Transporting $\psi$ by (4.15) is equivalent to move the boundary of $\Omega$ (the zero level-set of $\psi$ ) along the descent gradient direction $-J^{\prime}(\Omega)$.

\subsection{Application to the design of dielectric resonator filters}

This sub-section presents the application of shape optimization techniques (LS and TG) coupled with a finite element method for optimizing, in 3D, the shape of microwave components composed of ceramic objects in order to improve their electrical properties.

More precisely, these components are dielectric resonator filters and, traditionally, their design consists of optimizing the geometrical dimensions of resonators and coupling elements in order to achieve the desired function. One can use typical shapes of resonators and optimize their dimensions as a trade-off between quality factor and isolation (Fiedziusko \& Holme, 2001). The quality factor is related to the achievable insertion losses while the isolation is related to the stop-band characteristics. In order to relax this compromise, shape optimization methods (in our case TG and LS methods) can be utilized in order to improve a reference design obtained with a classical approach, i.e. based on a dielectric resonator with a regular shape.

\subsubsection{Improvement of unloaded quality factor}

A single pole filter can be constructed with a dielectric resonator inserted in metallic cavity and excited with two metallic irises connected to rectangular waveguides as shown in Figure 4.2. The unloaded quality factor of the dielectric resonator can be estimated by simulation or measurement of scattering parameters. Consequently, a shape optimization of the DR can be conducted for improving its unloaded quality factor.

Applying the level-set method, the unloaded $Q$ is increased by more than $50 \%$ as shown in Table 4.1. The optimal shape is depicted in Figure 4.2 (b). The dielectric resonator has been processed by ceramic stereolithography. The dielectric material is characterized by a relative permittivity $\left(\varepsilon_{\mathrm{r}}\right)$ equal to 9 and a loss tangent $(\tan \delta)$ equal to $4.510^{-5}$.

\begin{tabular}{|l|cc||}
\hline & Simulation & Measurement \\
\hline Reference resonator & 2000 & 900 \\
Optimized resonator & 3340 & 1910 \\
\hline
\end{tabular}

Table 4.1. Simulated and measured unloaded quality factors

\subsubsection{Improvement of stop-band performance}

A two-pole filter is derived from Figure 4.2(a) by cutting a corner in the dielectric resonator and rotating the output iris and waveguide as shown in Figure 4.3(a). Stop-band performance is altered by spurious transmissions due to higher-order modes.

For reducing the impact of spurious transmissions, the shape of the dielectric resonator is optimized applying the topology gradient technique in order to attenuate S21 parameter above the passband. The optimized resonator is shown in Figure 4.3(b) and scattering parameters obtained with reference and optimized resonators are compared in Figure 4.4. 


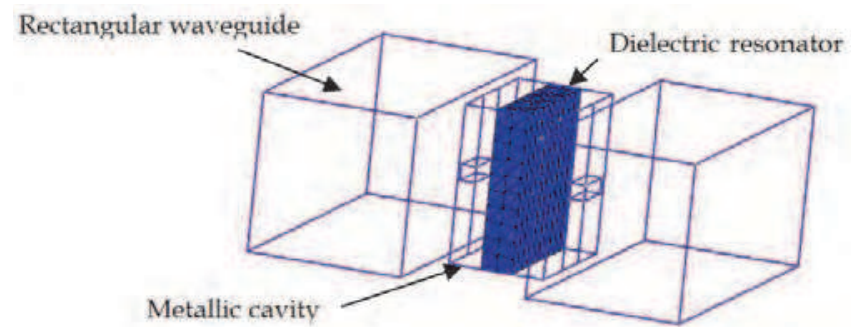

(a)

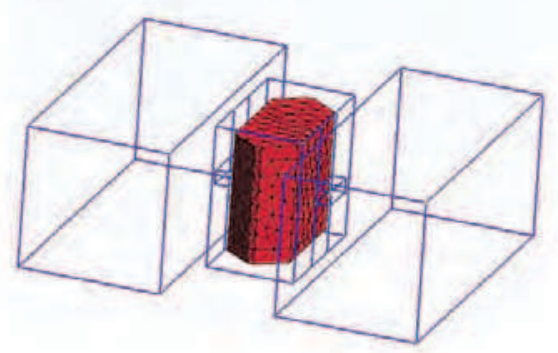

(b)

Fig. 4.2. Improvement of unloaded quality factor: (a) reference resonator, (b) optimized resonator

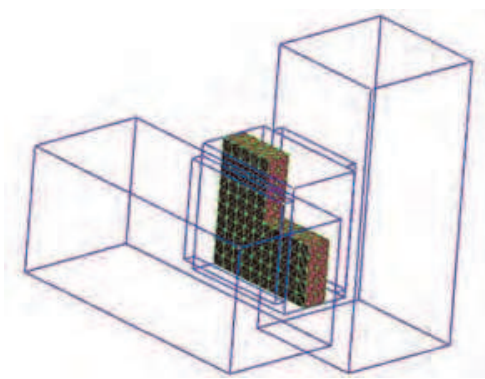

(a)

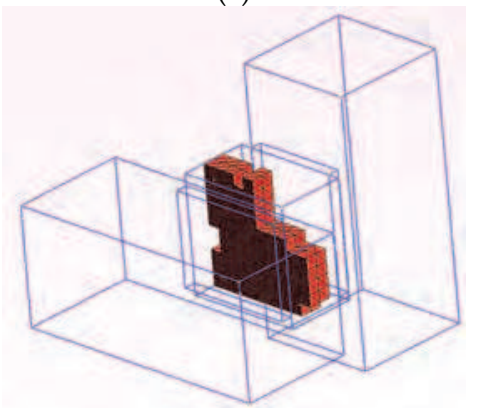

(b)

Fig. 4.3. Improvement of stop-band performance: (a) reference resonator, (b) optimized resonator 


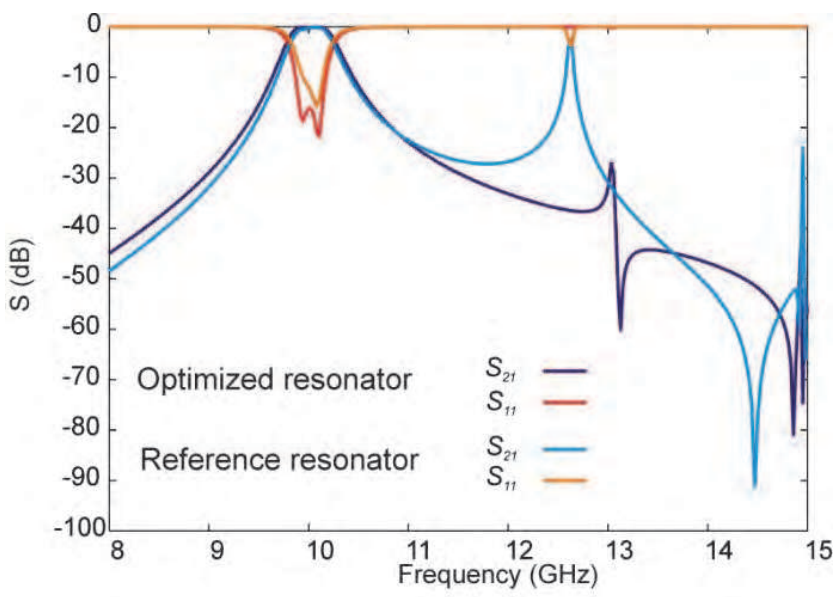

Fig. 4.4. Scattering parameters obtained by simulation of reference and optimized resonators.

\subsubsection{Design of dielectric resonator filters}

A four-pole dual-mode filter is constructed using two dual-mode resonators assembled together with a metallic cross-iris, as shown in Figure 4.5(a). One can optimize the functionality of the filter by adjusting the dimensions of both, the corners of the dual-mode resonators and the two arms of the metallic cross-iris.

A first shape-optimized design is conducted for replacing the metallic cross-iris by a ceramic coupling element in order to obtain a similar behavior in the pass-band and close out-ofband regions. Optimized resonator is shown in Figure 4.5(b) and scattering parameters are compared in Figure 4.6.

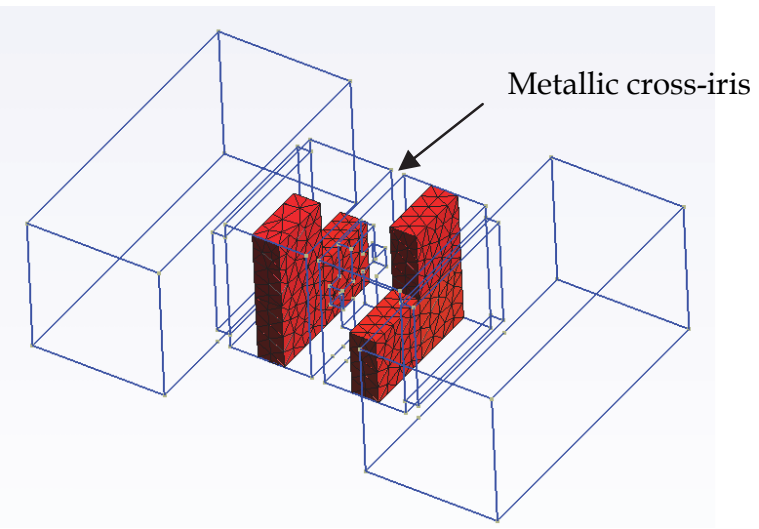

(a) 


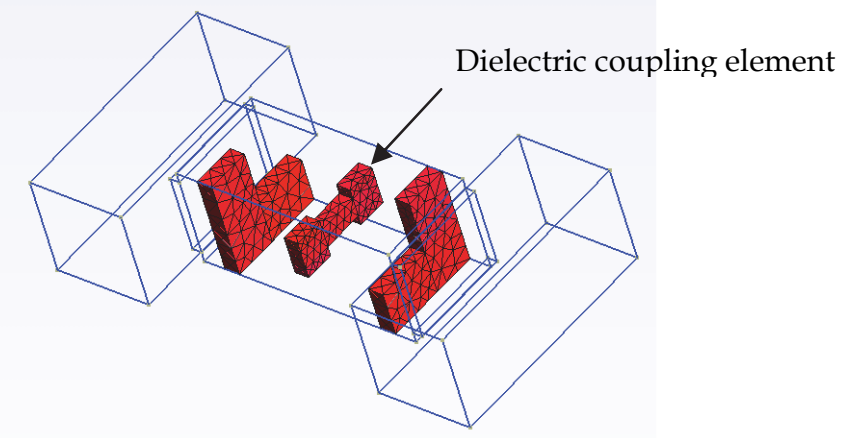

(b)

Fig. 4.5. Four-pole DR filter: (a) with a metallic cross-iris (reference) and (b) with a dielectric coupling element

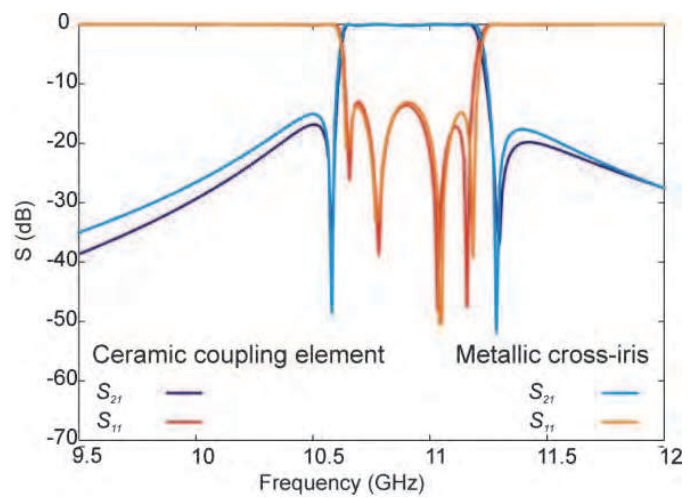

(a)

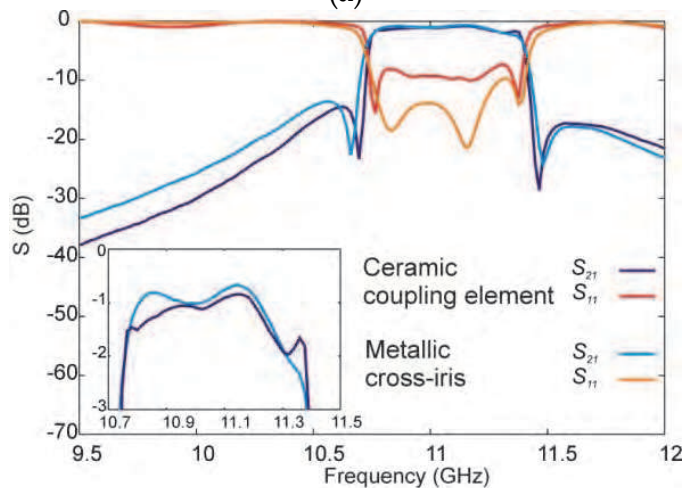

(b)

Fig. 4.6. S-parameters obtained by simulation (a) and measure (b) of reference and optimized coupling elements. 
A second design is performed on the basis of the previous optimization for improving the out-of-band performance of the filter. The initial and final resonators are shown in Figures 4.7(a) and 4.7(b). The simulation and measure of each resonator are compared in Figures 4.8(a) and 4.8(b).

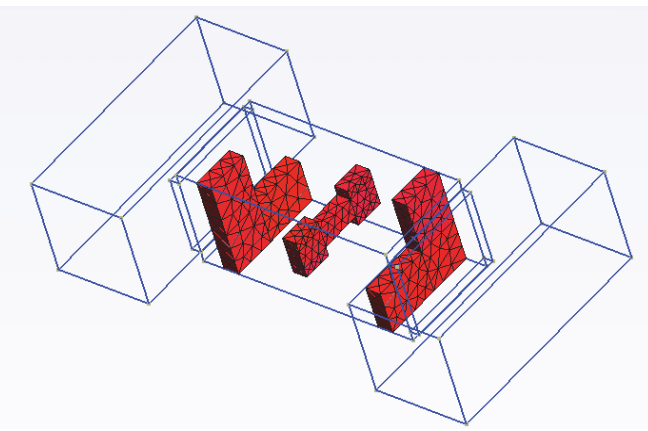

(a)

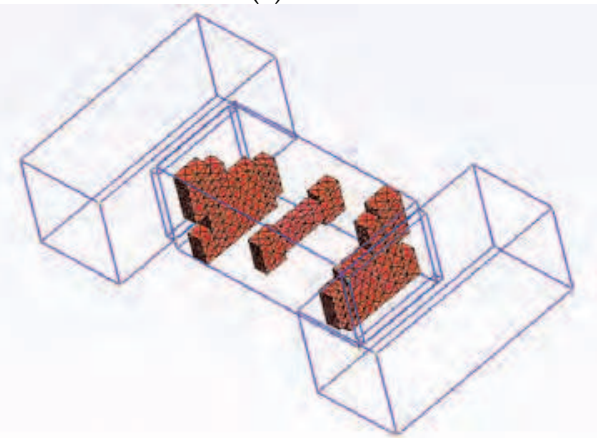

(b)

Fig. 4.7. Four-pole DR filter: (a) with reference resonators and (b) with optimized resonators.

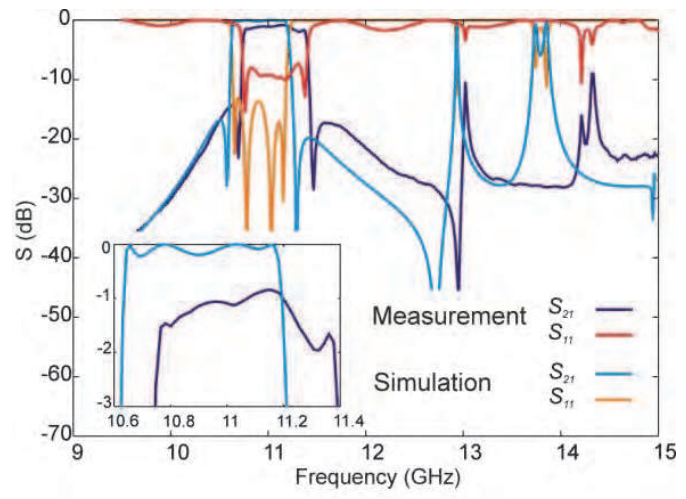

(a) 


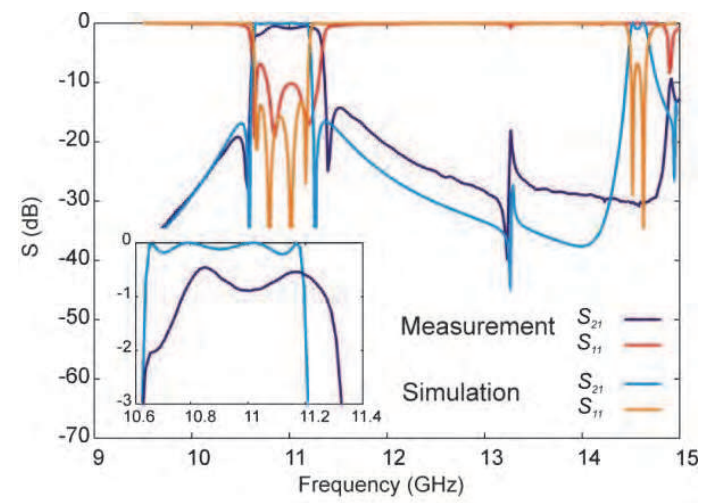

(b)

Fig. 4.8. S-parameters obtained by simulation and measure of reference (a) and optimized

(b) resonators

\section{Conclusion}

This chapter is focused on advanced design and fabrication techniques for ceramic-based microwave and millimeter-wave components. Complementary theoretical and fabrication approaches have been coupled in order to design innovative RF components successfully. As shown, the theoretical approaches are based on 3D electromagnetic and shape optimization methods whereas the rapid prototyping is based on 3D ceramic stereolithography process.

Moreover, the high electrical performances of these components and their geometrical and physical properties demonstrate that ceramic materials are suitable for such microwave applications with high constraints (geometrical, mechanical, thermal and electrical).

At last, this work proves that the $3 \mathrm{D}$ ceramic stereolithography process will be in the next future an interesting alternative technological process for the fabrication of 3-D monolithic all-dielectric devices at millimeter wavelengths.

\section{References}

Akel, H. \& Webb, J.P. (2000). Design sensitivities for scattering-matrix calculation with tetrahedral edge elements. IEEE Transactions on Magnetics, Vol. 36, No. 4, pp. 10431046, 2000.

Allaire, G. \& Jouve, F. (2002). Optimal design of micro-mechanisms by the homogenization method. European Journal of Finite Elements, Vol. 11, pp.405-416, 2002

Allaire, G.; De Gournay, F.; Jouve, F. \& Toader, A.M. (2003). Structural optimization using topological and shape sensitivity via a level set method. International Series of Numerical Mathematics, Vol. 147, pp.1-15, Birkhauser, Basel 2003.

Assadi-Haghi, A.; Bila, S.; Durousseau, C.; Baillargeat, D.; Aubourg, M.; Verdeyme, S.; Rochette, M.; Puech, J. \& Lapierre, L. (2006). Design of microwave components using topology gradient optimization. Proceedings of the 36th European Microwave Conference, pp. 462-465, 2006. 
Bertsch, A.; Jiguet, S., Renaud, P. (2003). Microfabrication of ceramic components by microstereolithography, J. Micromech., Vol. 14, pp. 197-203, 2003

Brakora, K. F.; Halloran, J.; Sarabandi, K. (2007). Design of 3-D monolithic MMW antennas using ceramic stereolithography. IEEE Trans. Antennas Propagat.,Vol. 55, No. 3, pp. 790-797, March 2007

Byun, J.K; Park, I.H.; Nah, W.; Lee, J.H. \& Kang, J. (2004). Comparison of shape and topology optimization methods for HTS solenoid design. IEEE Transactions on Applied Superconductivity, Vol. 14, No. 2, pp. 1842-1845, 2004

Chartier, T.; Chaput, C., Doreau, F., Loiseau, M. (2002). Stereolithography of structural complex ceramic parts, J. Mat. Sci., Vol. 37, pp. 3141-3147, 2002

Delhote, N.; Baillargeat, D.; Verdeyme, S.; Thevenot, M.; Delage, C. \& Chaput, C. (2007a). Large experimental bandpass waveguide in 3D EBG woodpile manufactured by layer-by-layer ceramic stereolithography. IEEE/MTT-S International Microwave Symposium, Honolulu, Hawaï, USA, pp 1431-1434, 2007

Delhote, N.; Baillargeat, D.; Verdeyme, S.; Delage, C.; Chaput, C.; Duterte, C.; Abouliatim, Y. \& Chartier, T. (2007b). Electromagnetic band gap millimeter and submillimeter 3D resonators manufactured by ceramic Stereolithography. Asia-Pacific Microwave Conference, Bangkok, Thaïlande, pp 1-4, 2007

Delhote ,N.; Baillargeat, D.; Verdeyme, S.; Delage, C. \& Chaput, C. (2007c). Ceramic layer-bylayer stereolithography for the manufacturing of $3 \mathrm{D}$ millimeter wave filters. IEEE Transactions on Microwave Theory and Techniques, Vol. 55, No. 3, pp. 548-554, 2007.

Delhote, N.; Baillargeat, D.; Verdeyme, S.; Delage, C. \& Chaput, C. (2007d). Innovative Shielded High Q Dielectric Resonator Made of Alumina By Layer-By-Layer Stereolithography. IEEE Microwave and Wireless Component Letters, volume 17, issue 6, pp 433-435, 2007.

Doreau, F.; Chaput, C.; Chartier, T. (2000). Stereolithography for manufacturing ceramic parts, Adv. Eng. Mater., Vol. 2, pp. 493-496, 2000

Fiedziuszko, J.S. \& Holme, S. (2001). Dielectric resonators raise your high-Q. IEEE Microwave Magazine, Vol. 2, No. 3, pp. 50-60, 2001.

Hinczewski, C. (1998). Ph. D., Institut National Polytechnique de Lorraine, France, 1998

Hull, C.; (1986). Apparatus for production of three dimensional objects by stereolithography, U.S. Pat. No. 4575 330, 1986

Khalil, A.H.; Delhote, N.; Pacchini S.; Claus, J.; Mardivirin, D.; Baillargeat, D.; Verdeyme, S.; Leblond, H. (2011). 3-D pyramidal and collective Ku band pass filters made in Alumina by ceramic stereolithography. IEEE/MTT-S International Microwave Symposium, Baltimore, USA, 2011

Khalil, H; Delhote, N.; Bila, S.; Aubourg, M.; Verdeyme, S.; Puech, J.; Lapierre, L.; Delage, C. \& Chartier, T. (2008). Topology optimization applied to the design of a dual-mode filter including a dielectric resonator. IEEE MTT-S International Microwave Symposium Digest, pp. 1381-1384, 2008.

Khalil, H.; Bila, S.; Aubourg, M.; Baillargeat, D.; Verdeyme, S.; Puech, J.; Lapierre, L.; Delage, C. \& Chartier, T. (2009). Topology optimization of microwave filters including dielectric resonators. Proceedings of the 39th European Microwave Conference, pp. 687690, 2009

Khalil, H.; Bila, S.; Aubourg, M.; Baillargeat, D.; Verdeyme, S.; Jouve, F.; Delage, C. \& Chartier, T. (2010). Shape optimized design of microwave dielectric resonators by 
level-set and topology gradient methods. International Journal of RF and Microwave Computer-Aided Engineering, Vol. 20, No. 1, pp 33-41, 2010

Khalil, H.; Bila, S.; Aubourg, M.; Baillargeat, D.; Verdeyme, S.; Jouve, F. \& Chartier, T. (2011). Shape optimization of dielectric resonators for improving their unloaded quality factor. International Journal of RF and Microwave Computer Aided Engineering, Vol. 21, No. 1, pp 102-126, 2011

Kim, Y.S.; Byun, J.K \& Park, I.H. (2009). A level set method for shape optimization of electromagnetic systems. IEEE Transaction on Magnetics, Vol. 45, No. 3, pp. 14661469, 2009.

Kozak, A. \& Gwarek, W. (1998). Unrestricted arbitrary shape optimization based on 3D electromagnetic simulation. IEEE MTT-S International Microwave Symposium Digest 1, pp. 17-20, 1998

Li, B.; Xu, Y. \& Choi, J. (1996). Applying Machine Learning Techniques, Proceedings of ASME 2010 4th International Conference on Energy Sustainability, pp. 14-17, ISBN 842-650823-3, Phoenix, Arizona, USA, May 17-22, 2010

Lima, P.; Bonarini, A. \& Mataric, M. (2004). Application of Machine Learning, InTech, ISBN 978-953-7619-34-3, Vienna, Austria

Liu, B.; Gong, X.; Chappell, W.J. (2004). Applications of layer-by-layer polymer stereolithography for three-dimensional high-frequency components. IEEE Trans. Microwave Theory Tech., Vol. 52, No. 11, pp. 2567-2575, November 2004

Mader, P.; Masmoudi, M. \& Mangenot, C. (2001). Topological asymptotic for waveguide optimization. IEEE Antennas and Propagation Society, AP-S International Symposium (Digest 1), pp 616-619, 2001.

Monneret, S. (2005). La microstereolithographie et ses applications," Méc. E Ind., Vol. 6, pp. 457-462, 2005

Nguyen, N.T.; Sauleau, R.; Delhote, N.; Baillargeat, D. \& Le Coq, L. (2010). Design and Characterization of $60-\mathrm{GHz}$ Integrated Lens AntennasFabricated Through Ceramic Stereolithography. IEEE Transactions on Antennas and Propagation, Vol. 58 No. 8 , pp $2757-2762,2010$.

Osher, J.S. \& Sethian, J.A. (1988). Fronts propagating with curvature dependent speed: algorithms based on Hamilton-Jacobi Formulations. Journal of Computational Physics, Vol. 79, No. 1, pp. 12-49, 1988.

Osher, J.S. \& Santosa, F. (2001). Level set method for optimization problems involving geometry and constraints I. Frequencies of a two-density inhomogeneous drum. Journal of Computational Physics, Vol. 171, pp. 272-288, 2001.

Sethian J.A. \& Wiegmann, A. (2000). Structural boundary design via level set and immersed interface methods. Journal of Computational Physics, Vol. 163, No. 2, pp. 489-528, 2000.

Sokolowski, J.S. \& Zochowski, A. (1999). On the topological derivative in shape optimization. SIAM Journal on Control and Optimization, Vol. 37, No. 4, pp. 12511272, 1999.

Suri, J.S.; Liu, K.; Singh, S.; Laxminarayan, S.N.; Zeng, X. \& Reden, L. (2002). Shape recovery algorithms using level sets in 2-D/3-D medical imagery - A state-of-the-art review. IEEE Transactions on Information Technology in Biomedicine, Vol. 6, No. 1, pp. 8-28, 2002. 


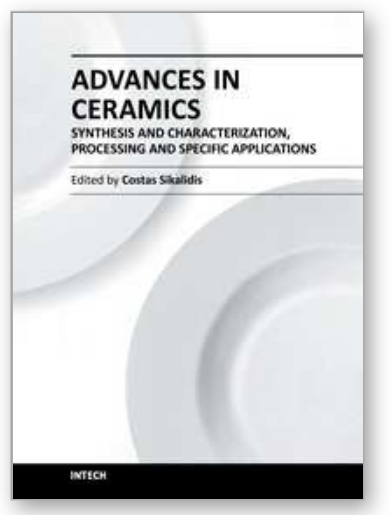

\author{
Advances in Ceramics - Synthesis and Characterization, \\ Processing and Specific Applications \\ Edited by Prof. Costas Sikalidis
}

ISBN 978-953-307-505-1

Hard cover, 520 pages

Publisher InTech

Published online 09, August, 2011

Published in print edition August, 2011

The current book contains twenty-two chapters and is divided into three sections. Section I consists of nine chapters which discuss synthesis through innovative as well as modified conventional techniques of certain advanced ceramics (e.g. target materials, high strength porous ceramics, optical and thermo-luminescent ceramics, ceramic powders and fibers) and their characterization using a combination of well known and advanced techniques. Section II is also composed of nine chapters, which are dealing with the aqueous processing of nitride ceramics, the shape and size optimization of ceramic components through design methodologies and manufacturing technologies, the sinterability and properties of $\mathrm{ZnNb}$ oxide ceramics, the grinding optimization, the redox behaviour of ceria based and related materials, the alloy reinforcement by ceramic particles addition, the sintering study through dihedral surface angle using AFM and the surface modification and properties induced by a laser beam in pressings of ceramic powders. Section III includes four chapters which are dealing with the deposition of ceramic powders for oxide fuel cells preparation, the perovskite type ceramics for solid fuel cells, the ceramics for laser applications and fabrication and the characterization and modeling of protonic ceramics.

\title{
How to reference
}

In order to correctly reference this scholarly work, feel free to copy and paste the following:

N. Delhote, S. Bila, D. Baillargeat, T Chartier and S Verdeyme (2011). Advanced Design and Fabrication of Microwave Components Based on Shape Optimization and 3D Ceramic Stereolithography Process, Advances in Ceramics - Synthesis and Characterization, Processing and Specific Applications, Prof. Costas Sikalidis (Ed.), ISBN: 978-953-307-505-1, InTech, Available from: http://www.intechopen.com/books/advances-inceramics-synthesis-and-characterization-processing-and-specific-applications/advanced-design-andfabrication-of-microwave-components-based-on-shape-optimization-and-3d-ceramic-s

\section{INTECH}

open science | open minds

\author{
InTech Europe \\ University Campus STeP Ri \\ Slavka Krautzeka 83/A \\ 51000 Rijeka, Croatia \\ Phone: +385 (51) 770447 \\ Fax: +385 (51) 686166
}

\author{
InTech China \\ Unit 405, Office Block, Hotel Equatorial Shanghai \\ No.65, Yan An Road (West), Shanghai, 200040, China \\ 中国上海市延安西路65号上海国际贵都大饭店办公楼 405 单元 \\ Phone: +86-21-62489820 \\ Fax: $+86-21-62489821$
}


www.intechopen.com 
(C) 2011 The Author(s). Licensee IntechOpen. This chapter is distributed under the terms of the Creative Commons Attribution-NonCommercialShareAlike-3.0 License, which permits use, distribution and reproduction for non-commercial purposes, provided the original is properly cited and derivative works building on this content are distributed under the same license. 